\title{
ON MODULAR FUNCTIONS IN CHARACTERISTIC $p$
}

\author{
BY
}

\author{
WEN-CH'ING WINNIE LI ${ }^{1}$
}

\begin{abstract}
Let $k=F_{q}(T)$ be a function field of one variable over a finite field $\mathbf{F}_{q}$. For a nonzero polynomial $A \in \mathrm{F}_{q}[T]$ one can define the modular group $\Gamma(A)$. In this paper, we continue a theme introduced by Weil, and study the $\lambda$-harmonic modular functions for $\Gamma(A)$. The main purpose of this paper is to give a natural definition of $\lambda$-harmonic Eisenstein series for $\Gamma(A)$ so that we obtain a decomposition theory of $\lambda$-harmonic modular functions, analogous to the classical results of Hecke. That is, we prove

Modular Functions $=$ Eisenstein Series $\oplus$ Cusp Functions.

Moreover, the dimension of the space generated by $\lambda$-harmonic Eisenstein series for $\Gamma(A)$ is equal to the number of cusps of $\Gamma(A)$, and so is independent of $\lambda$.

For the definition of $\lambda$-harmonic Eisenstein series and the proof of decomposition theory, we consider two cases: (i) $\lambda \neq \pm 2 \sqrt{q}$ and (ii) $\lambda= \pm 2 \sqrt{q}$, separately. Case (i) is treated in the usual way. Case (ii), being a "degenerate" case, is more interesting and requires more complicated analysis.
\end{abstract}

Introduction. Let $k=\mathrm{F}_{q}(T)$, where $\mathbf{F}_{q}$ is a finite field with $q$ elements and $T$ an indeterminant over $\mathbf{F}_{q}$. We write $\infty$ for the place of $k$ for which $|T|_{\infty}>1$. For a nonzero polynomial $A \in \mathbf{F}_{q}[T]$, let

$$
\begin{aligned}
\Gamma(A)=\left\{\left(\begin{array}{ll}
a & b \\
c & d
\end{array}\right) \in \operatorname{SL}\left(2, \mathbf{F}_{q}[T]\right) \mid\right. \\
a \equiv d \equiv 1(\bmod A), b \equiv c \equiv 0(\bmod A)\} .
\end{aligned}
$$

In this paper we continue a theme introduced by Weil [13], and study $\lambda$-harmonic modular functions for $\Gamma(A)$. (A modular function for $\Gamma(A)$ is said to be $\lambda$-harmonic if it is an eigenfunction of the Hecke operator $T_{\infty}$ with eigenvalue $\lambda$.) The main purpose of this paper is to give a natural definition of $\lambda$-harmonic Eisenstein series for $\Gamma(A)$ so that we obtain a decomposition theory of $\lambda$-harmonic modular functions, analogous to the classical results of Hecke [6, No. 24]. That is, we prove

Modular Functions $=$ Eisenstein Series $\oplus$ Cusp Functions.

Received by the editors May 28, 1976.

AMS (MOS) subject classifications (1970). Primary 10D05, 10D10.

${ }^{1}$ Research supported in part by the National Science Foundation.

(c) American Mathematical Society 1979 
Moreover, the dimension of the space generated by $\lambda$-harmonic Eisenstein series for $\Gamma(A)$ is equal to the number of cusps of $\Gamma(A)$, and so is independent of $\lambda$.

For $\lambda \neq \pm 2 \sqrt{q}$, such Eisenstein series are treated in the usual way, as in Hecke [6, No. 24] and Kubota [7]. Our analysis of these Eisenstein series will be carried out concretely in terms of graphs. In particular, when $A$ is a constant, this approach, in different language, is a special case of some results of Harder [5].

The most interesting cases are when $\lambda= \pm 2 \sqrt{q}$, because then the Eisenstein series obtained from the usual method of analytic continuation generate a space of dimension less than the number of cusps. As we shall see in $\S 3$, this is due to the "degeneracy" of the Eisenstein series so obtained. However, in this situation, the derivatives of these Eisenstein series are $\lambda$-harmonic and noncuspidal. It turns out that these derivatives exactly fill in the above deficiency. We therefore define these derivatives, in addition to those obtained from analytic continuation, to be Eisenstein series.

The author wishes to thank J.-P. Serre for his valuable advice on this paper.

\section{Preliminaries.}

1.1. The upper half-plane. Let $k_{\infty}$ be the completion of $k$ at $\infty$, and $r_{\infty}$ be the ring of integers of $k_{\infty}$. One may view the Poincaré upper half-plane as $\mathrm{GL}(2, \mathbf{R}) / 3_{\mathbf{R}} \cdot O(2, \mathbf{R})$, where $3_{\mathbf{R}}$ is the center of $\operatorname{GL}(2, \mathbf{R})$ and $O(2, \mathbf{R})$ the orthogonal subgroup of $G L(2, \mathbf{R})$. In the function field case, we take the corresponding "upper half-plane $\mathfrak{G}$ " to be the space of right cosets $\operatorname{GL}\left(2, k_{\infty}\right) / 3_{\infty} \cdot \operatorname{GL}\left(2, r_{\infty}\right)$, where $3_{\infty}$ is the center of $\operatorname{GL}\left(2, k_{\infty}\right)$, for the following reason:

For each row vector $(x, y)$ in $k_{\infty} \times k_{\infty}$, put $\operatorname{ht}(x, y)=\sup \left(|x|_{\infty},|y|_{\infty}\right)$. Then $\mathrm{GL}\left(2, r_{\infty}\right)$ is the group of linear transformations $r$ on $k_{\infty} \times k_{\infty}$ which preserve $\mathrm{ht}(x, y)$, i.e., $\mathrm{ht}((x, y) \cdot r)=\mathrm{ht}(x, y)$ for every $(x, y)$ in $k_{\infty} \times k_{\infty}$, and preserve the valuation of the determinant of elements in $\mathrm{GL}\left(2, k_{\infty}\right)$, i.e., $|\operatorname{det} g \cdot r|_{\infty}=|\operatorname{det} g|_{\infty}$ for every $g$ in $\operatorname{GL}\left(2, k_{\infty}\right)$, just as $O(2, \mathbf{R})$ preserves inner product on $\mathbf{R}^{2}$.

For short, we denote $\Gamma(1)$ by $\Gamma$. The group $\Gamma$ acts on $\mathscr{B}$ by left translations. For each integer $n$, put $\sigma_{n}=\left(\begin{array}{cc}T^{n} & 9 \\ 0 & 1\end{array}\right)$. Then every element $g$ of GL $\left(2, k_{\infty}\right)$ can be written as $g=\gamma \sigma_{n} g_{0}$ with $\gamma \in \Gamma, n \geqslant 0, g_{0} \in \mathrm{GL}\left(2, r_{\infty}\right) \cdot 3_{\infty}$. Moreover, when $g$ is given, the integer $n$ in this formula is uniquely determined. (For proof, see Weil [13].) Denote the coset $g \cdot \mathrm{GL}\left(2, r_{\infty}\right) \cdot 3_{\infty}$ by $[g]$. The upper half-plane is then $\mathscr{G}=\left\{\left[\gamma \sigma_{n}\right] \mid \gamma \in \Gamma, n \geqslant 0\right\}$. Since the integer $n$ in $\left[\gamma \sigma_{n}\right]$ is uniquely determined, call it the degree of $\left[\gamma \sigma_{n}\right]$. Two elements $\left[g_{1}\right],\left[g_{2}\right]$ in $\mathfrak{G}$ are said to be adjacent if there exist $\gamma \in \Gamma$ and nonnegative integers $m, n$ with $|m-n|=1$ such that $\left[g_{1}\right]=\left[\gamma \sigma_{m}\right]$ and $\left[g_{2}\right]=\left[\gamma \sigma_{n}\right]$. The stabilizer of $\left[\gamma \sigma_{n}\right]$ in $\Gamma$ is 


$$
\gamma \cdot\left\{\left(\begin{array}{cc}
a & b \\
0 & a^{-1}
\end{array}\right) \in \Gamma \mid a \in \mathbf{F}_{q}^{*}, b \in \mathbf{F}_{q}^{*}[T] \text { with } \operatorname{ord}_{T} b<n\right\} \cdot \gamma^{-1}
$$

if $n>1$; and $\gamma \cdot \operatorname{SL}\left(2, \mathrm{~F}_{q}\right) \gamma^{-1}$ if $n=0$ (cf. [13]). It follows that the neighbors of $\left[\gamma \sigma_{n}\right]$ are

$$
\begin{aligned}
& {\left[\gamma \sigma_{n+1}\right] \text { and }\left[\gamma \cdot\left(\begin{array}{cc}
1 & \xi T^{n} \\
0 & 1
\end{array}\right) \sigma_{n-1}\right], \xi \in \mathbf{F}_{q} \quad \text { if } n \geqslant 1 ;} \\
& {\left[\gamma\left(\begin{array}{ll}
0 & 1 \\
1 & 0
\end{array}\right) \sigma_{1}\right] \text { and }\left[\gamma \cdot\left(\begin{array}{cc}
1 & 0 \\
\xi & 1
\end{array}\right) \sigma_{1}\right], \xi \in \mathbf{F}_{q} \quad \text { if } n=0 .}
\end{aligned}
$$

In either case, we see that $\left[\gamma \sigma_{n}\right]$ has $q+1$ neighbors, namely,

$$
\left[\gamma \sigma_{n} \cdot \sigma_{1}\right] \text { and }\left[\gamma \sigma_{n} \cdot\left(\begin{array}{cc}
T^{-1} & \xi \\
0 & 1
\end{array}\right)\right], \xi \in \mathbf{F}_{q} \text {. }
$$

Taking each element in $\mathscr{B}$ to be a vertex and joining adjacent vertices, one can show that the resulting graph is a "tree" (cf. Serre [11]). Let $\mathfrak{G}$ be endowed with this tree structure.

1.2. Fundamental domain. From the above discussion one sees that a fundamental domain for $\Gamma$ in $\mathfrak{B}$ is a ray:

$$
\left[\gamma \sigma_{0}\right] \quad\left[\gamma \sigma_{1}\right] \quad\left[\gamma \sigma_{2}\right] \quad\left[\gamma \sigma_{3}\right]
$$

Let $A$ be a monic polynomial in $\mathbf{F}_{q}[T]$ with $\operatorname{ord}_{T} A=a>0$. If $\left[\gamma \sigma_{n}\right]$ is a vertex with degree $n \geqslant a$ and

$$
\left[\gamma\left(\begin{array}{cc}
1 & \xi T^{n} \\
0 & 1
\end{array}\right) \sigma_{n-1}\right]
$$

is one of its neighbors, then there is a polynomial $U$ in $\mathbf{F}_{q}[T], \operatorname{ord}_{T} U<n$, such that $U+\xi T^{n} \equiv 0 \bmod A$ and, hence,

$$
\left[\gamma\left(\begin{array}{cc}
1 & \xi T^{n} \\
0 & 1
\end{array}\right) \sigma_{n-1}\right]=\left[\gamma\left(\begin{array}{cc}
1 & \xi T^{n}+U \\
0 & 1
\end{array}\right) \sigma_{n+1}\right]=\left[\gamma_{A} \cdot \gamma \sigma_{n-1}\right]
$$

for some $\gamma_{A} \in \Gamma(A)$ since $\Gamma(A)$ is normal and $\left(\begin{array}{c}1 \\ 0\end{array} T_{1}^{n}+U\right) \in \Gamma(A)$. This proves part of the following:

LEMMA 1. If $n>a$, then all of the degree $n-1$ neighbors of $\left[\gamma \sigma_{n}\right]$ are in the same orbit of $\Gamma(A)$. If $n<a$, then no two neighbors of $\left[\gamma \sigma_{n}\right]$ are in the same orbit of $\Gamma(A)$.

Proof. It is obvious that no two elements of $\mathfrak{B}$ of distinct degree can lie in the same orbit of $\Gamma$. Only two cases are left to prove: 
Case I. $1 \leqslant n<a$. Suppose $\xi_{1}, \xi_{2} \in \mathbf{F}_{q}$ such that

$$
\left[\gamma\left(\begin{array}{cc}
1 & \xi_{1} T^{n} \\
0 & 1
\end{array}\right) \cdot \sigma_{n-1}\right] \text { and }\left[\gamma\left(\begin{array}{cc}
1 & \xi_{2} T^{n} \\
0 & 1
\end{array}\right) \sigma_{n-1}\right]
$$

are in the same orbit of $\Gamma(A)$. We claim $\xi_{1}=\xi_{2}$. The assumption means that there exists $\gamma_{A} \in \Gamma(A)$ such that

$$
\left(\begin{array}{cc}
1 & -\xi_{1} T^{n} \\
0 & 1
\end{array}\right) \gamma^{-1} \cdot \gamma_{A} \cdot \gamma \cdot\left(\begin{array}{cc}
1 & \xi_{2} T^{n} \\
0 & 1
\end{array}\right) \in \text { Stabilizer of }\left[\sigma_{n-1}\right] .
$$

Since $\Gamma(A)$ is normal, this amounts to saying

$$
\begin{aligned}
\gamma_{A}^{\prime} \cdot\left(\begin{array}{cc}
1 & -\xi_{1} T^{n} \\
0 & 1
\end{array}\right) \gamma^{-1} \gamma \cdot\left(\begin{array}{cc}
1 & \xi_{2} T^{n} \\
0 & 1
\end{array}\right) & =\gamma_{A}^{\prime} \cdot\left(\begin{array}{cc}
1 & \left(\xi_{2}-\xi_{1}\right) T^{n} \\
0 & 1
\end{array}\right) \\
& \in \text { Stabilizer of }\left[\sigma_{n-1}\right],
\end{aligned}
$$

for some $\gamma_{A}^{\prime} \in \Gamma(A)$. Write $\gamma_{A}^{\prime}=\left(\begin{array}{c}P \\ R\end{array}\right)$. Then

$$
\begin{aligned}
\gamma_{A}^{\prime}\left(\begin{array}{cc}
1 & \left(\xi_{2}-\xi_{1}\right) T^{n} \\
0 & 1
\end{array}\right) & =\left(\begin{array}{ll}
P & Q \\
R & S
\end{array}\right)\left(\begin{array}{cc}
1 & \left(\xi_{2}-\xi_{1}\right) T^{n} \\
0 & 1
\end{array}\right) \\
& =\left(\begin{array}{cc}
P & P\left(\xi_{2}-\xi_{1}\right) T^{n}+Q \\
R & *
\end{array}\right) \in \text { Stabilizer of }\left[\sigma_{n-1}\right]
\end{aligned}
$$

implies $P, R \in \mathbf{F}_{q}, P\left(\xi_{2}-\xi_{1}\right) T^{n}+Q \in \mathbf{F}_{q}[T]$ of order less than $n$, hence $Q=0, P \neq 0$, and $\xi_{1}=\xi_{2}$ because $Q \equiv 0 \bmod A$ and $n<a$.

Case II. $n=0$. The neighbors of $\left[\gamma \sigma_{0}\right]$ are $\left[\gamma\left(\begin{array}{cc}0 & 1 \\ 1 & 0\end{array}\right) \sigma_{1}\right]$ and $\left[\gamma\left(\begin{array}{ll}1 & 0 \\ \xi & 1\end{array}\right) \sigma_{1}\right], \xi \in \in \mathbf{F}_{q}$. Assume $g_{1}, g_{2}$ are two elements in $\mathrm{GL}\left(2, \mathrm{~F}_{q}\right)$ such that $\left[\gamma g_{1} \sigma_{1}\right]=\left[\gamma_{A} \gamma g_{2} \sigma_{1}\right]$ for some $\gamma_{A} \in \Gamma(A)$, i.e.,

$$
\gamma_{A}^{\prime} \cdot g_{1}^{-1} g_{2} \in \text { Stabilizer of }\left[\sigma_{1}\right]
$$

for some $\gamma_{A}^{\prime} \in \Gamma(A)$. Write

$$
\gamma_{A}^{\prime}=\left(\begin{array}{ll}
P & Q \\
R & S
\end{array}\right) \text { and } g_{1}^{-1} g_{2}=\left(\begin{array}{ll}
x & y \\
u & v
\end{array}\right)
$$

then

$$
\gamma_{A}^{\prime} \cdot g_{1}^{-1} g_{2}=\left(\begin{array}{cc}
* & * \\
x R+S u & *
\end{array}\right) .
$$

As discussed before, every element in the stabilizer of $\left[\sigma_{1}\right]$ is upper triangular, therefore $x R+S u=0$; in particular, $x R+S u \equiv u \equiv 0(\bmod A)$. This shows that $u=0$, i.e. $g_{1}^{-1} g_{2}$ is upper triangular. However, if $g_{1}, g_{2}$ are two distinct elements of

$$
\left\{\left(\begin{array}{ll}
0 & 1 \\
1 & 0
\end{array}\right),\left(\begin{array}{ll}
1 & 0 \\
\xi & 1
\end{array}\right) \mid \xi \in \mathbf{F}_{q}\right\}
$$


$g_{1}^{-1} g_{2}$ is never upper triangular. Therefore no two neighbors of $\left[\gamma \sigma_{0}\right]$ are in the same orbit of $\Gamma(A)$. Q.E.D.

Let

$$
\gamma_{i}=\left(\begin{array}{ll}
P_{i} & Q_{i} \\
R_{i} & S_{i}
\end{array}\right) \in \Gamma, \quad i=1, \ldots, N,
$$

be left coset representatives of $\Gamma(A)$ in $\Gamma$. We choose $\gamma_{i}$ 's in such a way that the orders of $P_{i}, Q_{i}, R_{i}, S_{i}$ are less than $a+1$. Then a fundamental domain $\mathscr{D}(A)$ for $\Gamma(A)$ can be obtained by gluing the rays

$$
\left[\gamma_{i} \overline{\left.\sigma_{0}\right]}\left[\gamma_{i} \sigma_{1}\right] \quad\left[\gamma_{i} \sigma_{2}\right] \quad\left[\gamma_{i} \sigma_{3}^{-}\right]\right.
$$

together at the identical vertices. Lemma 1 shows that each vertex of degree $<a-1$ has exactly $q+1$ neighbors, each of multiplicity one, but each vertex of degree $n \geqslant a$ has one degree $n-1$ neighbor with multiplicity $q$ and one degree $n+1$ neighbor with multiplicity one. The rays

$$
\left[\gamma_{i} \sigma_{a-1}\right] \quad\left[\gamma_{i} \sigma_{a}\right] \quad\left[\gamma_{i} \sigma_{a+1}\right] \quad \cdots \cdots
$$

are called "cusps" of $\Gamma(A)$ determined by $\gamma_{i}$. (For more details, see Cartier [1]). It is clear that two elements

$$
\gamma=\left(\begin{array}{ll}
P & Q \\
R & S
\end{array}\right), \quad \gamma^{\prime}=\left(\begin{array}{ll}
P^{\prime} & Q^{\prime} \\
R^{\prime} & S^{\prime}
\end{array}\right)
$$

in $\Gamma$ determine the same cusp of $\Gamma(A)$ if and only if there is some $\alpha \in \mathrm{F}_{q}^{*}$ such that $P \equiv \alpha P^{\prime}$ and $R \equiv \alpha R^{\prime}$ mod $A$. With this understanding, we shall denote cusps of $\Gamma(A)$ simply by $\left(\begin{array}{l}P \\ R\end{array}\right)$. As in the classical case, the number of cusps of $\Gamma(A)$ is

$$
\sigma(A)=\left\{\begin{array}{l}
\frac{1}{q-1} \cdot|A|_{\infty}^{2} \cdot \prod_{\substack{B \text { monic polynomial } \\
B \text { divides } A}}\left(1-\frac{1}{|B|_{\infty}^{2}}\right) \text { if ord } A=a>1, \\
1 \text { if } A=1 .
\end{array}\right.
$$

It is also clear that, in $\mathscr{D}(A)$, there are $\sigma(A)$ vertices of degree $m \geqslant a-$ $1 ; \sigma(A) \cdot q^{a-1-m}$ vertices of degree $m, 1<m<a-1$; and $\sigma(A) \cdot$ $q^{a-1} /(q+1)$ vertices of degree 0 . One can refer to $\$ 4$ for examples.

1.3. Modular forms and modular functions. From now on let $A$ be a fixed monic polynomial in $\mathrm{F}_{q}[T]$ with $\operatorname{ord}_{T} A=a>0$.

Definition. Let $s$ be a complex number. A homogeneous modular form $F$ for $\Gamma(A)$ of weight $s$ is a complex-valued function defined on $\operatorname{GL}\left(2, k_{\infty}\right)$, left 
invariant under $\Gamma(A)$, right invariant under $\mathrm{GL}\left(2, r_{\infty}\right)$, which satisfies

$$
F\left(g \cdot\left(\begin{array}{ll}
a & 0 \\
0 & a
\end{array}\right)\right)=|a|_{\infty}^{-s} F(g)
$$

for all $g \in \mathrm{GL}\left(2, k_{\infty}\right)$ and all $\left(\begin{array}{cc}a & 0 \\ 0 & a\end{array}\right) \in 3_{\infty}$.

It is well known that any element $g \in \mathrm{GL}\left(2, k_{\infty}\right)$ can be written as

$$
g=\left(\begin{array}{cc}
T^{n} & y \\
0 & 1
\end{array}\right) \cdot \delta \cdot\left(\begin{array}{cc}
T^{l} & 0 \\
0 & T^{l}
\end{array}\right)
$$

where $\delta \in \mathrm{GL}\left(2, r_{\infty}\right), y \in k_{\infty}$. If

$$
g=\left(\begin{array}{cc}
T^{n} & y \\
0 & 1
\end{array}\right) \delta\left(\begin{array}{cc}
T^{l} & 0 \\
0 & T^{l}
\end{array}\right)=\left(\begin{array}{cc}
T^{m} & y^{\prime} \\
0 & 1
\end{array}\right) \delta^{\prime}\left(\begin{array}{cc}
T^{l^{\prime}} & 0 \\
0 & T^{l^{\prime}}
\end{array}\right)
$$

with $y, y^{\prime} \in k_{\infty}, \delta, \delta^{\prime} \in \mathrm{GL}\left(2, r_{\infty}\right)$, then

$$
\begin{gathered}
\left(\begin{array}{cc}
T^{-l} & 0 \\
0 & T^{-l}
\end{array}\right)\left(\begin{array}{cc}
T^{-n} & -y T^{-n} \\
0 & 1
\end{array}\right)\left(\begin{array}{cc}
T^{m} & y^{\prime} \\
0 & 1
\end{array}\right)\left(\begin{array}{cc}
T^{l^{\prime}} & 0 \\
0 & T^{l^{\prime}}
\end{array}\right) \\
=\left(\begin{array}{cc}
T^{m-n-l+l^{\prime}} & T^{-n-l+l^{\prime}}\left(y^{\prime}-y\right) \\
0 & T^{l^{\prime}-l}
\end{array}\right) \in \mathrm{GL}\left(2, r_{\infty}\right)
\end{gathered}
$$

implies $l=l^{\prime}, m=n$, and $y^{\prime}-y \in T^{n} r_{\infty}$. Therefore, if we write

$$
g=\left(\begin{array}{cc}
T^{n} & y \\
0 & 1
\end{array}\right) \delta\left(\begin{array}{cc}
T^{l} & 0 \\
0 & T^{l}
\end{array}\right)
$$

with $\delta \in \mathrm{GL}\left(2, r_{\infty}\right)$ and $y=T^{n+1} h, h \in \mathbf{F}_{q}[T]$, then this expression is unique. Noting this, we can now define the dehomogenization $f$ of a homogeneous modular form $F$ of weight $s$ for $\Gamma(A)$ to be the function on $\$$ which sends $[g]$ to $F\left(\left(\begin{array}{cc}T^{n} & y\end{array}\right)\right)$ where $g$ is uniquely expressed by

$$
g=\left(\begin{array}{cc}
T^{n} & y \\
0 & 1
\end{array}\right) \cdot \delta\left(\begin{array}{cc}
T^{l} & 0 \\
0 & T^{l}
\end{array}\right)
$$

as described above. One can easily check that for

$$
\left(\begin{array}{ll}
P & Q \\
R & S
\end{array}\right) \in \Gamma(A) \text { and }\left(\begin{array}{ll}
x & y \\
u & v
\end{array}\right) \in G L\left(2, k_{\infty}\right)
$$

$f$ satisfies

$$
f\left(\left[\left(\begin{array}{ll}
P & Q \\
R & S
\end{array}\right)\left(\begin{array}{ll}
x & y \\
u & v
\end{array}\right)\right]\right)=\frac{\mathrm{ht}\left((R, S)\left(\begin{array}{ll}
x & y \\
u & v
\end{array}\right)\right)^{s}}{\mathrm{ht}(u, v)^{s}} \cdot f\left(\left[\begin{array}{ll}
x & y \\
u & v
\end{array}\right]\right) .
$$

A complex-valued function on $\mathfrak{G}$ which satisfies $(1.1)$ for all $\left({ }_{R}^{P} \xi\right) \in \Gamma(A)$ and $\left(\begin{array}{ll}x & y \\ u & v\end{array}\right) \in \mathrm{GL}\left(2, k_{\infty}\right)$ is called a modular form for $\Gamma(A)$ of weight $s$. When $s=0$, we say it is a modular function. Note that the function $G_{0}$ which sends $g$ in $\mathrm{GL}\left(2, k_{\infty}\right)$ to $|\operatorname{det} g|_{\infty}^{-s / 2}$ is a homogeneous modular form of weight $s$ for the 
modular group $\Gamma$. Therefore one can transform a homogeneous modular form for $\Gamma(A)$ of weight $s$ to a modular function for $\Gamma(A)$ (or conversely) by dividing (or multiplying) by $G_{0}$. Hence it suffices to understand modular functions.

For a complex-valued function $f$ on $\mathscr{B}$, the Hecke operator $T_{\infty}$ maps $f$ to another function $T_{\infty} f$ on $\mathfrak{B}$ defined by

$$
\begin{aligned}
T_{\infty} f([g]) & =f\left(\left[g \sigma_{1}\right]\right)+\sum_{\xi \in \mathbf{F}_{q}} f\left(\left[g \cdot\left(\begin{array}{cc}
T^{-1} & \xi \\
0 & 1
\end{array}\right)\right]\right) \\
& =\sum_{\substack{\left[g_{1}\right] \text { adjacent } \\
\text { to }[g]}} f\left(\left[g_{1}\right]\right) .
\end{aligned}
$$

$f$ is said to be $\lambda$-harmonic if $f$ is an eigenfunction of $T_{\infty}$ with eigenvalue $\lambda$.

In fact, $T_{\infty}$ here can be interpreted as the Beltrami operator. (For more detail, see Weil [14, Chapter VIII].) In this paper, we are interested in $\lambda$-harmonic modular functions only.

REMARK. Technically speaking, modular forms defined above are analogous to modular forms $f$ of weight $s$ defined on the Poincare upper half-plane which satisfy

$$
f\left(\frac{a \tau+b}{c \tau+d}\right)=|c \tau+d|^{s} f(\tau)
$$

for $\tau$ in the complex upper half-plane and $\left(\begin{array}{ll}a & b \\ c & d\end{array}\right)$ in some modular subgroup of $\operatorname{SL}(2, Z)$. The $\lambda$-harmonic modular functions defined above correspond, in the classical case, to the "real analytic" modular functions in the sense of Kubota [7], namely, complex-valued functions on the Poincare upper halfplane which are eigenfunctions of the Laplacian $y^{2}\left(\partial^{2} / \partial x^{2}+\partial^{2} / \partial y^{2}\right)$, and which are invariant under linear transformations by a modular group. So it is not surprising to find that the techniques which we use later are similar to those used in [7].

Let $f$ be a $\lambda$-harmonic modular function for $\Gamma(A)$. First observe that, on each cusp, $f$ satisfies the difference equation

$$
f\left(\left[\gamma \sigma_{n+2}\right]\right)-\lambda f\left(\left[\gamma \sigma_{n+1}\right]\right)+q f\left(\left[\gamma \sigma_{n}\right]\right)=0 \text { for all } n \geqslant a-1 .
$$

Write the roots of $x^{2}-\lambda x+q=0$ as $q^{s / 2}, q^{1-s / 2}$ where $s$ is a complex number with $\operatorname{Re} s>1$, and is uniquely determined modulo $4 \pi i / \log q$ if $\operatorname{Re} s>1$. Then for each cusp $\left(\begin{array}{l}P \\ R\end{array}\right)$ determined by some $\gamma \in \Gamma$ there exist two constants $a_{(P, R)}, b_{(P, R)} \in \mathbf{C}$ such that for all $n \geqslant a-1$,

$$
f\left(\left[\gamma \sigma_{n}\right]\right)=\left\{\begin{array}{ll}
a_{(P, R)} q^{n \cdot s / 2}+b_{(P, R)} q^{n(1-s / 2)} & \text { if } q^{s / 2} \neq q^{1-s / 2} \\
a_{(P, R)} q^{n \cdot s / 2}+b_{(P, R)} \cdot q^{n \cdot s / 2} & \text { if } q^{s / 2}=q^{1-s / 2}= \pm q^{1 / 2}
\end{array} .\right.
$$

Call $a_{(P, R)}\left(\right.$ resp. $\left.b_{(P, R)}\right)$ the $q^{n \cdot s / 2}$ (resp. $\left.q^{n(1-s / 2)}\right)$ part of $f$ on the cusp $\left(\begin{array}{l}P \\ R\end{array}\right)$ if 
$q^{s / 2} \neq q^{1-s / 2}$. In case $q^{s / 2}=q^{1-s / 2}$, we call $a_{(P, R)}\left(\right.$ resp. $\left.b_{(P, R)}\right)$ the $q^{n \cdot s / 2}$ (resp. $n q^{s / 2}$ ) part of $f$ on the cusp $\left(\begin{array}{l}P \\ R\end{array}\right)$. It is clear that a $\lambda$-harmonic modular function vanishes on the cusp $\left(\begin{array}{l}P \\ R\end{array}\right)$ if and only if both its $q^{n \cdot s / 2}$ part and its $q^{n(1-s / 2)}$ part (or $n \cdot q^{n \cdot s / 2}$ part if $\lambda= \pm 2 \sqrt{q}$ ) are zero on the cusp $\left(\begin{array}{l}P \\ R\end{array}\right)$.

The group $\Gamma$ acts on a modular function $f$ for $\Gamma(A)$ by left translations, namely, $\gamma \cdot f([g])=f([\gamma g)]$ for all $\gamma \in \Gamma,[g] \in \mathfrak{B}$. Note that $\gamma \cdot f$ is also a modular function for $\Gamma(A)$ since $\Gamma(A)$ is a normal subgroup of $\Gamma$. Moreover, if $f$ is $\lambda$-harmonic, so is $\gamma f$.

1.4. Fourier expansions of modular functions. Let $f$ be a modular function for $\Gamma(A)$, and

$$
H_{n}=\left\{\left[\left(\begin{array}{cc}
T^{n} & y \\
0 & 1
\end{array}\right)\right] \mid y \in k_{\infty}\right\} .
$$

Then $\mathfrak{B}=\cup_{n=-\infty}^{\infty} H_{n}$ and $H_{n}$ are called horocycles. The restriction of $f$ to $H_{n}$ can be viewed as a function on $k_{\infty}$, invariant under translations by $A \cdot \mathbf{F}_{q}[T]$, therefore can be expanded in a Fourier series on the compact group $k_{\infty} / A$. $\mathbf{F}_{q}[T]$. Take a fixed nontrivial character $\varphi$ of the additive group of $\mathbf{F}_{q}$. For $y \in k_{\infty}$, there exist unique $x \in \mathrm{F}_{q}[T], z \in T^{a-1} r_{\infty}$ such that $y=A \cdot x+z$. Write $z=\alpha_{a-1} T^{a-1}+$ terms of lower order. Define the character $\psi$ of $k_{\infty}$, trivial on $A \cdot \mathbf{F}_{q}[T]$, by $\psi(y)=\varphi\left(\alpha_{a-1}\right)$ where $y, \alpha_{a-1}$ are as above. Then all the characters of $k_{\infty}$ are of the form $\psi_{d}: y \rightarrow \psi(d y), d \in k_{\infty}$; and those trivial on $A \cdot \mathbf{F}_{q}[T]$ are exactly $\psi_{Q}$ with $Q \in \mathbf{F}_{q}[T]$. Let $d y$ denote the Haar measure on $k_{\infty}$ with $\operatorname{vol}\left(T^{a-1} \cdot r_{\infty}\right)=1$. Thus, for all $\left[\left(\begin{array}{cc}T^{n} & y \\ 0 & 1\end{array}\right)\right]$ in $H_{n}$,

$$
f\left(\left[\left(\begin{array}{cc}
T^{n} & y \\
0 & 1
\end{array}\right)\right]\right)=\sum_{Q \in \mathbf{F}_{q}[T]} C(n, Q) \psi_{Q}(y)
$$

where

$$
C(n, Q)=\int_{A \cdot \mathbf{F}_{q}[T] \backslash k_{\infty}} f\left(\left[\left(\begin{array}{cc}
T^{n} & y \\
0 & 1
\end{array}\right)\right]\right) \psi_{-Q}(y) d y .
$$

Since

$$
\left[\left(\begin{array}{cc}
T^{n} & y \\
0 & 1
\end{array}\right)\right]=\left[\left(\begin{array}{cc}
T^{n} & 0 \\
0 & 1
\end{array}\right)\right] \text { if } y \in T^{n} r_{\infty}
$$

it follows that $C(n, Q)=0$ if $n+\operatorname{ord}_{T} Q>a-1$, so the above summation is in fact a finite sum. If $n \geqslant a-1$, then

$$
f\left(\left[\left(\begin{array}{cc}
T^{n} & y \\
0 & 1
\end{array}\right)\right]\right)=f\left(\left[\left(\begin{array}{cc}
T^{n} & 0 \\
0 & 1
\end{array}\right)\right]\right)=C(n, 0) .
$$

When $C(n, 0)=0$ for all $n \in \mathbf{Z}$, we say that $f$ is $\left(\begin{array}{l}1 \\ 0\end{array}\right)$-cuspidal. For $\gamma=\left(\begin{array}{l}P \\ R\end{array}\right)$ $\in \Gamma$ we say that $f$ is $\left(\begin{array}{l}P \\ R\end{array}\right)$-cuspidal if the modular function $\gamma \cdot f$ is $\left(\begin{array}{l}1 \\ 0\end{array}\right)$-cuspidal. A 
modular function for $\Gamma(A)$ is cuspidal if it is $\left({ }_{R}^{P}\right)$-cuspidal for all cusps $\left({ }_{R}^{P}\right)$ of $\Gamma(A)$.

It can easily be shown (cf. Weil [13]) that a modular function $f$ for $\Gamma(A)$ is $\lambda$-harmonic if and only if its Fourier coefficients $C(n, Q)$ satisfy

$$
\lambda C(n, Q)=C(n+1, Q)+C(n-1, Q) \cdot \sum_{\xi \in \mathbf{F}_{q}} \psi_{Q}\left(\xi T^{n}\right)
$$

for all $Q \in \mathbf{F}_{q}[T]$ and all $n \in \mathbf{Z}$. As an immediate consequence of this, one sees that a $\lambda$-harmonic modular function is cuspidal if and only if it vanishes at each cusp.

2. Eisenstein series and decomposition theory (Part I: $\lambda \neq \pm 2 \sqrt{q}$ ).

2.1. Eisenstein series. As before, let $A$ be a fixed monic polynomial in $\mathbf{F}_{q}[T]$ with $\operatorname{ord}_{T} A=a>0$. We make the following conventions: for $X, Y$ in $\mathrm{F}_{q}[T]$ $X \equiv Y$ means $X \equiv Y(\bmod A) ;(X, Y)$ denotes the monic polynomial in $F_{q}[T]$ of highest order which divides both $X$ and $Y$. Following Weil [13], for $s \in \mathbf{C}, P, R \in \mathbf{F}_{q}[T]$ with $(P, R, A)=1$, we define the Eisenstein series $E_{(P, R)}(s)$ on $\mathfrak{B}$ by

$$
\begin{aligned}
E_{(P, R)}(s)([g]) & =|\operatorname{det} g|_{\infty}^{s / 2} \sum_{\substack{X \equiv P \\
Y \equiv R \\
(X, Y)=1}} \operatorname{ht}((X, Y) g)^{-s} \\
& =|\operatorname{det} g|_{\infty}^{s / 2} \sum_{\gamma \in \operatorname{St}(P, R) \backslash \Gamma(A)} \operatorname{ht}((P, R) \gamma g)^{-s}
\end{aligned}
$$

where $\operatorname{St}(P, R)=\{\gamma \in \Gamma(A) \mid(P, R) \gamma=(P, R)\}$. As explained in $\S 1.3$, these are analogous to classical homogenized Eisenstein series of weight " $s$ " if we omit the factor $|\operatorname{det} g|_{\infty}^{s / 2}$.

It is obvious that $E_{(P, R)}(s)$ defined by $(2.1)$ is invariant under $\Gamma(\mathrm{A})$, hence on each horocycle $H_{n}, E_{(P, R)}(s)$ has Fourier series expansion

$$
E_{(P, R)}\left(\left[\left(\begin{array}{cc}
T^{n} & y \\
0 & 1
\end{array}\right)\right], s\right)=\sum_{Q \in \mathrm{F}_{q}[T]} C_{(P, R)}(n, Q, s) \psi_{Q}(y) .
$$

A direct computation shows that

$$
\begin{gathered}
C_{(P, R)}(n, 0, s)=q^{n(1-s / 2)} \cdot q^{1-a} \cdot \Phi_{(P, R)}(0, s)+\delta_{P, A} \delta_{R, 1} \varepsilon \cdot q^{n \cdot s / 2}, \\
C_{(P, R)}(n, Q, s)=0 \text { if } Q \neq 0, \text { and } n>a-2-\text { ord } Q, \\
C_{(P, R)}(a-2-\text { ord } Q-d, Q, s)=q^{(a-2-\text { ord } Q-d)(1-s / 2)} \\
\cdot \Phi_{(P, R)}(Q, s) \cdot \frac{1-q^{(d+1)(1-s)}}{1-q^{1-s}} \cdot q^{1-a}
\end{gathered}
$$


if $Q \neq 0$ and $d>0$, where

$$
\begin{aligned}
\delta_{P, A} & = \begin{cases}1 & \text { if } P \equiv 0 \bmod A, \\
0 & \text { otherwise; }\end{cases} \\
\delta_{R, 1} & = \begin{cases}1 & \text { if } R \equiv \text { some } \alpha \text { in } \mathrm{F}_{q}^{*}, \\
0 & \text { otherwise; }\end{cases} \\
\varepsilon & = \begin{cases}1 & \text { if } \operatorname{ord}_{T} A>1, \\
q-1 & \text { if } \operatorname{ord}_{T} A=0 ;\end{cases} \\
\Phi_{(P, R)}(0, s) & =\sum_{\substack{X \equiv P \\
X \neq 0}}|X|_{\infty}^{-s} \cdot \sum_{\substack{Y \equiv R \\
Y \text { mod } A X \\
(X, Y)=1}} 1 \cdot \frac{1-q^{-s}}{1-q^{1-s}} ;
\end{aligned}
$$

and

$$
\Phi_{(P, R)}(Q, s)=\sum_{\substack{X \equiv P \\ X \neq 0}}|X|_{\infty}^{-s} \cdot \sum_{\substack{Y \equiv R \\ Y \text { mod } A X \\(X, Y)=1}} \psi_{Q}\left(\frac{Y}{X}\right) \cdot\left(1-q^{-s}\right) .
$$

Using the Euler $\varphi$-function

$$
\varphi(A)=|A|_{\infty} \prod_{\substack{B \text { monic prime } \\ B \mid A}}\left(1-|B|_{\infty}^{-1}\right)
$$

and the $L$-series

$$
L(\chi, s)=\prod_{B \text { monic prime }}\left(1-\chi(B)|B|_{\infty}^{-s}\right)^{-1}
$$

associated to each character $\chi$ on $\mathbf{F}_{q}[T] \bmod A$, one can further express (2.6) and (2.7) as

$$
\begin{aligned}
\Phi_{(P, R)}(0, s)= & (q-1) \cdot q^{a(1-s)} \frac{1-q^{-s}}{1-q^{2-s}} \cdot \prod_{B \text { prime }}\left(1-|B|_{\infty}^{-s}\right)^{-1} \\
& +\frac{1}{\varphi(A)} \cdot \frac{1-q^{-s}}{1-q^{1-s}} \cdot \sum_{\substack{\alpha \bmod A \\
(\alpha, A)=1}} \widetilde{\left|\alpha^{-1} P\right|_{\infty}^{1-s}} \\
& \cdot \sum_{\chi \text { char mod } A} \bar{\chi}(\alpha) \cdot L(\chi, s)^{-1}
\end{aligned}
$$


and

$$
\begin{aligned}
\Phi_{(P, R)}(Q, s)= & \frac{1-q^{-s}}{\varphi(A)} \cdot \sum_{\substack{\alpha \bmod A \\
(\alpha, A)=1}} \sum_{\substack{X \mid Q \\
\alpha X \equiv P}}|X|_{\infty}^{1-s} \cdot \psi_{Q}\left(\widetilde{\frac{\alpha^{-1} R}{X}}\right) \\
& \cdot \sum_{\chi c h a r \bmod A} \bar{\chi}(\alpha) \cdot L(\chi, s)^{-1}
\end{aligned}
$$

where, for each $\alpha \in F_{q}[T]$ with $(\alpha, A)=1, \widetilde{\alpha^{-1} P}$ denotes the unique polynomial in $F_{q}[T]$ with order less than $a$ such that $\alpha \cdot \overparen{\alpha}-\alpha_{P} \equiv P \bmod A$. It is understood that $\left|\alpha^{-1} P\right|_{\infty}^{1-s}$ denotes the zero function as $A$ divides $P$. Since $L(\chi, s)$ is a rational function in $q^{-s}$ (cf. Weil [12]) and $L(\chi, s)$ does not vanish on $\operatorname{Re} s>1$ (by a similar argument as in Lang [8, Chapter XV, Theorems 2, 3]) for each character $\chi \bmod A$, the functions $\Phi_{(P, R)}(Q, s), Q \neq 0$, defined by (2.9) are rational functions in $q^{-s}$ and holomorphic on the half-plane $\operatorname{Re} s>$ 1.

LEMMA 2. Let $P, R \in \mathrm{F}_{q}[T]$ with $(P, R, A)=1$. The function $\Phi_{(P, R)}(0, s)$ defined by (2.8) is a rational function in $q^{-s}$ and is holomorphic on $\operatorname{Re} s>1$ except for simple poles at $s=2+2 n \pi i / \log q, n \in \mathbf{Z}$.

Proof. It is clear that for $\operatorname{Re} s>1, \Phi_{(P, R)}(0, s)$ is holomorphic except for simple poles at $s=2+2 n \pi i / \log q, n \in \mathbf{Z}$. It remains to prove that $\Phi_{(P, R)}(0, s)$ does not have a pole on the line $\operatorname{Re} s=1$. The first term in the right-hand side of (2.8) is clearly holomorphic on the line $\operatorname{Re} s=1$, so it suffices to check that for each character $\chi \bmod A$, and each integer $n$,

$$
\lim _{s \rightarrow 1+2 \pi n i / \log q} \frac{1}{1-q^{1-s}} \cdot \sum_{\substack{\alpha \bmod A \\(\alpha, A)=1}}\left|\widetilde{\alpha^{-1} P}\right|_{\infty}^{1-s} \bar{\chi}(\alpha) \cdot L(\chi, s)^{-1}
$$

is finite if $\boldsymbol{A}$ does not divide $P$.

Case I. $\chi=\chi_{1}$, the trivial character. Since

$$
L\left(\chi_{1}, s\right)=\left(1-q^{1-s}\right)^{-1} \cdot \prod_{\substack{B \text { prime } \\ B \mid A}}\left(1-|B|_{\infty}^{-s}\right),
$$

(2.10) becomes

$$
\begin{aligned}
\lim _{s \rightarrow 1+2 \pi n i / \log q} \frac{1}{1-q^{1-s}} \cdot & \left.\sum_{\substack{\alpha \bmod A \\
(\alpha, A)=1}} \widetilde{\mid \alpha-1 P}\right|_{\infty} ^{1-s} \\
& \cdot\left(1-q^{1-s}\right) \cdot \prod_{\substack{B \text { prime } \\
B \mid A}}\left(1-|B|_{\infty}^{-s}\right)^{-1}=|A|_{\infty}=q^{a},
\end{aligned}
$$

which is finite. 
Case II. $\chi \neq \chi_{1}$. Then $L(\chi, 1+2 \pi n i / \log q) \neq 0,(2.12)$ is equal to

$$
\begin{gathered}
\frac{1}{L(\chi, 1+2 \pi n i / \log q)} \lim _{s \rightarrow 1+2 \pi n i / \log q} \frac{1}{1-q^{1-s}} \cdot \sum_{\substack{\alpha \bmod A \\
(\alpha, A)=1}} q^{(1-s) \operatorname{ord} \alpha^{-1} P} \cdot \bar{\chi}(\alpha) \\
=\frac{1}{L(\chi, 1+2 \pi n i / \log q)} \lim _{u \rightarrow 1} \frac{1}{1-u} \sum_{\substack{\alpha \bmod A \\
(\alpha, A)=1}} u^{\text {ord } \alpha^{-1} P} \bar{\chi}(\alpha) \\
=\frac{1}{L(\chi, 1+2 \pi n i / \log q)} \lim _{u \rightarrow 1}-\sum_{\substack{\alpha \bmod A \\
(\alpha, A)=1}} \text { ord } \widetilde{\alpha^{-1} P} \cdot u^{\text {ord } \widetilde{\alpha}^{-1} P-1} \bar{\chi}(\alpha) \\
=\frac{-1}{L(\chi, 1+2 \pi n i / \log q)} \sum_{\substack{\alpha \bmod A \\
(\alpha, A)=1}}^{\text {ord } \alpha^{-1 P} \cdot \bar{\chi}(\alpha),}
\end{gathered}
$$

which is also finite. Q.E.D.

For each $\left[\left(\begin{array}{cc}T^{n} y \\ 1\end{array}\right)\right] \in \mathfrak{G}$, define $E_{(P, R)}(s)\left(\left[\left(T_{0}^{n} y_{1}\right)\right]\right)$ by $(2.2)$ where $C_{(P, R)}(n, 0, s)$ is defined by (2.3); $C_{(P, R)}(n, Q, s), Q \neq 0$, are defined by (2.4) and (2.5) with $\Phi_{(P, R)}(0, s)$ and $\Phi_{(P, R)}(Q, s), Q \neq 0$, defined by (2.8) and (2.9), respectively.

TheOREM 1. Let $P, R \in \mathrm{F}_{q}[T]$ with $(P, R, A)=1$. For fixed $[g] \in \mathfrak{g}$, the function $E_{(P, R)}(s)([g])$ defined above is a rational function in $q^{-s / 2}$, and is holomorphic on $\operatorname{Re} s \geqslant 1$ except for simple poles at $s=2+2 n \pi i / \log q, n \in \mathbf{Z}$. When $\operatorname{Re} s>2$, it is equal to (2.1). Moreover, the following properties hold:

(i)

$$
E_{(P, R)}(s)\left(\left[\gamma_{A} g\right]\right)=E_{(P, R)}(s)([g]) \text { for all } \gamma_{A} \in \Gamma(A),[g] \in \mathfrak{B}
$$

(ii)

$$
\begin{aligned}
& \left(q^{s / 2}+q^{1-s / 2}\right) E_{(P, R)}(s)([g]) \\
& \quad=E_{(P, R)}(s)([g])+\sum_{\xi \in \mathbf{F}_{q}} E_{(P, R)}(s)\left(\left[g \cdot\left(\begin{array}{cc}
T^{-1} & \xi \\
0 & 1
\end{array}\right)\right]\right)
\end{aligned}
$$

for all $[g] \in \mathfrak{B}$

(iii)

$$
E_{(P, R)}(s)=E_{\left(P^{\prime}, R^{\prime}\right)}(s) \text { if } P \equiv \alpha P^{\prime} \text { and } R \equiv \alpha R^{\prime} \text { for some } \alpha \in \mathrm{F}_{q}^{*} ;
$$

$$
\gamma E_{(P, R)}(s)=E_{(P, R)_{\gamma}}(s) \text { for all } \gamma \in \Gamma \text {, }
$$

for all $s \in \mathbf{C}$.

Proof. The first statement follows immediately from the definition of $E_{(P, R)}(s)$, Lemma 2, and the discussion above Lemma 2 . One can easily check that, for fixed $[g] \in \mathfrak{B}$, properties (i) to (iv) hold for $\operatorname{Re} s>2$. Since $E_{(P, R)}(s)([g])$ 's are meromorphic functions on $\mathbf{C}$ with a discrete set of poles, 
properties (i) to (iv) thus hold for all $s \in$ C. Q.E.D.

Theorem 1 shows that for $\operatorname{Re} s \geqslant 1, s \neq 2+2 n \pi i / \log q, n \in \mathbf{Z}$, the functions $E_{(P, R)}(s)$ are $\lambda$-harmonic modular functions for $\Gamma(A)$ with $\lambda=q^{s / 2}$ $+q^{1-s / 2}$. For $s \neq 2+2 n \pi i / \log q, 1+2 n \pi i / \log q$, $\operatorname{Re} s>1$, let $\mathcal{E}(\Gamma(A)$, $\left.q^{s / 2}+q^{1-s / 2}\right)$ be the space generated by all $E_{(P, R)}(s)$. The functions $\tilde{E}_{(P, R)}(s)$ $=E_{(P, R)}(s)-E_{(A, 1)}(s)$ are holomorphic on $\operatorname{Re} s>1$, and satisfy (i), (ii), (iii) of Theorem 1 with $E_{(P, R)}(s)$ replaced by $\tilde{E}_{(P, R)}(s)$, and

(iv)'

$$
\gamma \tilde{E}_{(P, R)}(s)=\tilde{E}_{(P, R) \gamma}(s)-\tilde{E}_{(A, 1) \gamma}(s) \text { for all } \gamma \in \Gamma,
$$

for all $s \in \mathrm{C}$. Thus, when $s=2+2 n \pi i / \log q, \tilde{E}_{(P, R)}(s)$ are $\lambda$-harmonic modular functions for $\Gamma(A)$ with $\lambda=q^{s / 2}+q^{1-s / 2}=q+1$ or $-(q+1)$ according as $n$ is even or odd. Furthermore, the constant functions are $(q+1)$-harmonic; and the alternating constant functions which take value $d$ on odd degree elements of $\mathscr{B}$ and $-d$ on even degree elements of $\mathscr{G}$ are $-(q+1)$-harmonic functions for $\Gamma$. Thus for $s=2+2 n \pi i / \log q, n$ even integer (resp. $n$ odd integer), $\lambda=q^{s / 2}+q^{1-s / 2}=q+1$ (resp. $\lambda=-(q+$ 1)), we let $\mathcal{E}\left(\Gamma(A), q^{s / 2}+q^{1-s / 2}\right)$ be the space generated by the constant functions (resp. the alternating constant functions) and all $\tilde{E}_{(P, R)}(s)$. The space $\mathcal{E}\left(\Gamma(A), q^{s / 2}+q^{1-s / 2}\right)$ defined above is called the space of $\left(q^{s / 2}+\right.$ $q^{1-s / 2}$ )-harmonic Eisenstein series for $\Gamma(A)$. Therefore we have defined the space of $\lambda$-harmonic Eisenstein series for $\Gamma(A)$ with $\lambda \neq \pm 2 \sqrt{q}$.

THEOREM 2. The space $\mathcal{E}(\Gamma(A), \lambda), \lambda \neq \pm 2 \sqrt{q}$ has dimension equal to $\sigma(A)$, the number of cusps of $\Gamma(A)$.

Proof. Write $\lambda=q^{s / 2}+q^{1-s / 2}$ for some $s$ with $\operatorname{Re} s \geqslant 1$. Let

$$
\left(\begin{array}{c}
P_{i} \\
R_{i}
\end{array}\right), \quad i=1, \ldots, \sigma(A)
$$

be cusps of $\Gamma(A)$.

Case I. $\lambda \neq \pm 2 \sqrt{q}, \pm(q+1)$. Theorem 1(iii) implies $\operatorname{dim}_{\mathbf{C}} \mathcal{E}(\Gamma(A), \lambda) \leqslant$ $\sigma(A)$. (2.3) and Theorem 1(iv) together show that $E_{\left(P_{i}, R_{i}\right)}(s)$ is the only one among the $E_{\left(P_{j}, R_{j}\right)}(s), j=1, \ldots, \sigma(A)$, which has nonzero $q^{n \cdot s / 2}$ part on the cusp $\left(\bar{P}_{i} R_{i}\right)$. This implies that $E_{\left(P_{i}, R_{i}\right)}, i=1, \ldots, \sigma(A)$, are linearly independent, hence $\operatorname{dim}_{\mathrm{C}} \mathcal{E}(\Gamma(A), \lambda)=\sigma(A)$.

Case II. $s=2+2 n \pi i / \log q$ for some $n \in \mathbf{Z}$, i.e., $\lambda=q+1$ or $-(q+1)$. It is obvious that the space generated by $\tilde{E}_{(P, R)}(s)$ has dimension at most equal to $\sigma(A)-1$ and therefore $\operatorname{dim}_{\mathrm{C}} \delta(\Gamma(A), \lambda) \leqslant \sigma(A)$. Suppose $\left(P_{R_{1}}^{P_{1}}\right)=$ $\left(\begin{array}{l}A \\ 1\end{array}\right)$. Let $E$ be a nonvanishing constant function, or a nonzero alternating constant function according as $\lambda=q+1$ or $-(q+1)$. Then on each cusp $E$ has zero $q^{n \cdot s / 2}$ part and nonzero $q^{n(1-s / 2)}$ part. Now claim that $E, \tilde{E}_{\left(P_{i}, R_{i}\right)}$, $i=2, \ldots, \sigma(A)$, are linearly independent. If so, we shall have 
$\operatorname{dim}_{\mathbf{C}} \mathcal{E}(\Gamma(A), \lambda)=\sigma(A)$. Suppose $\alpha_{1}, \ldots, \alpha_{\sigma(A)}$ are in $\mathbf{C}$ such that

$$
\alpha_{1} E+\sum_{i=2}^{\sigma(A)} \alpha_{i} \tilde{E}_{\left(P_{i}, R_{i}\right)}=0
$$

Let $\tilde{C}_{\left(P_{i}, R_{i}\right)}(n, Q, s)$ be the Fourier coefficients of $\tilde{E}_{\left(P_{i}, R_{i}\right)}(s)$. Then we have, in particular,

$$
\tilde{C}_{\left(P_{i}, R_{i}\right)}(n, 0, s)=q^{n(1-s / 2)} q^{1-a}\left(\Phi_{\left(P_{i}, R_{i}\right)}(0, s)-\Phi_{(A, 1)}(0, s)\right)-\varepsilon q^{n \cdot s / 2}
$$

for $i=2, \ldots, \sigma(A)$ and all $n \in \mathbf{Z}$. As explained above, for $i=2, \ldots, \sigma(A)$,

$$
\gamma \tilde{E}_{\left(P_{i}, R_{i}\right)}(s)=E_{\left(P_{i}, R_{i}\right) \gamma}(s)-E_{(A, 1) \gamma}(s) \text { for all } \gamma \in \Gamma \text {. }
$$

It then follows that $\tilde{E}_{\left(P_{i}, R_{i}\right)}(s)$ has nonzero $q^{n s / 2}$ part $\varepsilon,-\varepsilon$ on the cusps $\left({ }_{P_{i}} R_{i}\right)$, $\left(\begin{array}{l}1 \\ 0\end{array}\right)$ respectively; and has zero $q^{n s / 2}$ part on the other cusps. This shows $\alpha_{i}=0$ for $i=2, \ldots, \sigma(A)$, and hence $\alpha_{1}=0$, as desired. Q.E.D.

Finally, for each complex number $\lambda$, we let $\mathfrak{N}(\Gamma(A), \lambda)$ (resp. $\mathcal{C}(\Gamma(A), \lambda))$ be the space of $\lambda$-harmonic modular functions (resp. cusp functions) for $\Gamma(A)$.

2.2. Decomposition theory. Let $\mathscr{D}(A)$ be a fundamental domain of $\Gamma(A)$, and $\mathscr{F}(A)$ be the subset of $\mathscr{D}(A)$ consisting of elements in $\mathscr{D}(A)$ of degree $\leqslant a-1$, i.e., the finite part of $\mathscr{D}(A)$. For modular functions $F, G$ of $\Gamma(A)$, we define the restricted inner product $\langle,\rangle_{\mathscr{F ( A )}}$ by

$$
\langle F, G\rangle_{\mathscr{F}(A)}=\sum_{[g] \in \mathscr{F}(A)} F([g]) \overline{G([g])}
$$

where - means complex conjugation. It is understood that when $\operatorname{deg} A=a$ $=0, \mathscr{F}(A)$ is empty and $\langle F, G\rangle_{\mathscr{F}(A)}=0$ for all modular functions $F, G$ of $\Gamma(A)$. Similarly, the inner product $\langle$,$\rangle of two modular functions F, G$ for $\Gamma(A)$ is defined by

$$
\langle F, G\rangle=\sum_{[g] \in \mathscr{D}(A)} F([g]) \overline{G([g])}
$$

whenever the right-hand side converges absolutely. It is very interesting that almost all important consequences in our theory come from the following:

THEOREM 3. Let $\gamma_{1}, \ldots, \gamma_{o(A)}$ be elements in $\Gamma$ which determine the cusps of $\Gamma(A)$. Let $F, G$ be modular functions for $\Gamma(A), T_{\infty}$ the Hecke operator at $\infty$. Then

$$
\begin{aligned}
\left\langle F, T_{\infty} \bar{G}\right\rangle_{\mathscr{F}(A)}-\left\langle T_{\infty} F, \bar{G}\right\rangle_{\mathscr{F}(A)} \\
\quad=\sum_{i=1}^{\sigma(A)} F\left(\left[\gamma_{i} \sigma_{a-1}\right]\right) G\left(\left[\gamma_{i} \sigma_{a}\right]\right)-F\left(\left[\gamma_{i} \sigma_{a}\right]\right) G\left(\left[\gamma_{i} \sigma_{a-1}\right]\right) .
\end{aligned}
$$


Proof. From Lemma 1, we know that every element $[g]$ in $\mathscr{F}(A)$ has exactly $q+1$ distinct neighbors in $\mathscr{D}(A)$, each of multiplicity one. Therefore

$$
\begin{aligned}
\left\langle F, T_{\infty} \bar{G}\right\rangle_{\mathscr{F}(A)}-\left\langle T_{\infty} F, \bar{G}\right\rangle_{\mathscr{F}(A)} & =\sum_{\substack{[g] \in \mathscr{F}(A)\\
}} F([g]) \cdot \sum_{\substack{\left[g_{1}\right] \text { adjacent } \\
\text { to }[g]}} G\left(\left[g_{1}\right]\right)-\sum_{[g] \in \mathscr{F}(A)} G([g]) \\
& \cdot \sum_{\substack{\left[g_{1}\right] \text { adjacent } \\
\text { to }[g]}} F\left(\left[g_{1}\right]\right) \\
= & \sum_{\substack{[g],\left[g_{1}\right] \text { in } \mathscr{F}(A) \\
[g] \text { adjacent to }\left[g_{1}\right]}} F([g]) G\left(\left[g_{1}\right]\right)-F\left(\left[g_{1}\right]\right) G([g]) \\
& +\sum_{i=1}^{\sigma(A)} F\left(\left[\gamma_{i} \sigma_{a-1}\right]\right) G\left(\left[\gamma_{i} \sigma_{a}\right]\right)-\sum_{i=1}^{\sigma(A)} G\left(\left[\gamma_{i} \sigma_{a-1}\right]\right) F\left(\left[\gamma_{i} \sigma_{a}\right]\right) \\
= & \sum_{i=1}^{\sigma(A)} F\left(\left[\gamma_{i} \sigma_{a-1}\right]\right) G\left(\left[\gamma_{i} \sigma_{a}\right]\right)-G\left(\left[\gamma_{i} \sigma_{a-1}\right]\right) F\left(\left[\gamma_{i} \sigma_{a}\right]\right) . \quad \text { Q.E.D. }
\end{aligned}
$$

Corollary 1. Let $\gamma_{1}, \ldots, \gamma_{\sigma(A)}$ be as in Theorem $3, F, F^{\prime} \in \mathfrak{M}(\Gamma(A), \lambda)$ with $\lambda \neq \pm 2 \sqrt{q}$. Write $\lambda=q^{s / 2}+q^{1-s / 2}$ for some $s$ with $\operatorname{Re} s>1$. Suppose that on each cusp determined by $\gamma_{i}, i=1, \ldots, \sigma(A)$,

$$
\begin{gathered}
F\left(\left[\gamma_{i} \sigma_{m}\right]\right)= \begin{cases}a_{i} q^{m s / 2}+b_{i} q^{m(1-s / 2)} & \text { if } s \neq 2+2 n \pi i / \log q, \\
a_{i} q^{m s / 2}+b_{i} m q^{m s / 2} & \text { if } s=2+2 n \pi i / \log q,\end{cases} \\
F^{\prime}\left(\left[\gamma_{i} \sigma_{m}\right]\right)= \begin{cases}a_{i}^{\prime} q^{m s / 2}+b_{i}^{\prime} q^{m(1-s / 2)} & \text { if } s \neq 2+2 n \pi i / \log q, \\
a_{i}^{\prime} q^{m s / 2}+b_{i}^{\prime} m q^{m s / 2} & \text { if } s=2+2 n \pi i / \log q,\end{cases}
\end{gathered}
$$

for all $m \geqslant a-1$. Then

$$
\sum_{i=1}^{\sigma(A)} a_{i} b_{i}^{\prime}-a_{i}^{\prime} b_{i}=0 .
$$

Proof. $F^{\prime} \in \mathscr{T}(\Gamma(A), \lambda)$ implies $\bar{F}^{\prime} \in \mathscr{T}(\Gamma(A), \bar{\lambda})$. Then

$$
\left\langle F, T_{\infty} \bar{F}^{\prime}\right\rangle_{\mathscr{F}(A)}-\left\langle T_{\infty} F, \bar{F}^{\prime}\right\rangle_{\mathscr{F}(A)}=\lambda\left\langle F, \bar{F}^{\prime}\right\rangle_{\mathscr{F}(A)}-\lambda\left\langle F, \bar{F}^{\prime}\right\rangle_{\mathscr{F}(A)}=0 .
$$

Applying (2.11) to $F, F^{\prime},(2.12)$ follows immediately. Q.E.D.

THEOREM 4. The space $\mathcal{C}(\Gamma(A))$ of all cusp functions for $\Gamma(A)$ is finitedimensional. The Hecke operator $T_{\infty}$ is a selfadjoint operator on $\mathcal{C}(\Gamma(A))$ relative to the inner product $\langle$,$\rangle defined above, and, hence, \mathcal{C}(\Gamma(A))=$ $\bigoplus_{\lambda} \mathcal{C}(\Gamma(A), \lambda)$, where the $\lambda$ 's are real algebraic integers with $|\lambda|<q+1$. 
Proof. Let $F$ belong to $\mathcal{C}(\Gamma(A)), C(n, Q)$ be the Fourier coefficients of $F$ and $C^{\prime}(n, Q)$ be those of $T_{\infty} F$. Since

$$
C^{\prime}(n, Q)=C(n+1, Q)+C(n-1, Q) \cdot \sum_{\xi \in \mathbf{F}_{q}} \psi_{Q}\left(\xi T^{n}\right),
$$

it follows immediately that $T_{\infty}$ sends $\mathcal{C}(\Gamma(A))$ into itself. Note that the inner product $\langle$,$\rangle is defined on \mathcal{C}(\Gamma(A))$ and agrees with the restricted inner product $\langle,\rangle_{\mathscr{F}(A)}$. (2.11) shows that $\left\langle F, T_{\infty} G\right\rangle=\left\langle T_{\infty} F, G\right\rangle$ for all $F, G$ in $\mathcal{C}(\Gamma(A))$, i.e., $T_{\infty}$ is selfadjoint in $\mathcal{C}(\Gamma(A))$ and eigenvalues of $T_{\infty}$ on $\mathcal{C}(\Gamma(A))$ are real. It is obvious that the dimension of $\mathcal{C}(\Gamma(A))$ is less than the number of elements $[g]$ in $\mathscr{D}(A)$ with degree $\leqslant a-2$, which is finite, say $N$; and hence $\mathcal{C}(\Gamma(A))$ is the direct sum of eigenspaces $\mathcal{C}(\Gamma(A), \lambda)$ of $T_{\infty}$. If $0 \neq F \in$ $\mathcal{C}(\Gamma(A), \lambda)$, one may view $F$ as a nontrivial solution of a system of $K$ linear equations in $N$ variables, where $K$ is the cardinality of $\mathscr{F}(A)$. Expressing these relations by a $K \times N$ matrix $M=\left(m_{i j}\right)$, we see that $m_{i i}=\lambda$ for $i=1, \ldots, N$ and $m_{i j}=-1$ or 0 if $i \neq j$. Since $M$ has a nontrivial solution $F$, the rank of $M$ is less than $N$, i.e., $\lambda$ satisfies a monic polynomial of degree $N$ with integral coefficients, hence is an algebraic integer. Finally, by the maximal modulus principle, one finds that the only bounded functions on $\mathscr{B}, \lambda$-harmonic with $|\lambda|>q+1$ are constant functions and alternating constant functions on $\mathfrak{G}$ with $\lambda=q+1$ and $-(q+1)$, respectively. Therefore nontrivial $\lambda$-harmonic cusp functions for $\Gamma(A)$ must have $|\lambda|<q+1$. Q.E.D.

Lemma 3. Let $\gamma_{1}, \ldots, \gamma_{o(A)}$ be as in Theorem 3. Suppose $F \in \mathfrak{N}(\Gamma(A), \lambda)$, $\lambda \neq \pm 2 \sqrt{q}, \pm(q+1)$. Write $\lambda=q^{s / 2}+q^{1-s / 2}$ for some $s$ with $\operatorname{Re} s>1$. If at each cusp determined by $\gamma_{i}, F\left(\left[\gamma_{i} \sigma_{m}\right]\right)=b_{i} q^{m(1-s / 2)}$ for all $m>a-1$, then $F$ is cuspidal.

Proof. Suppose

$$
\gamma_{i}=\left(\begin{array}{cc}
P_{i} & Q_{i} \\
R_{i} & S_{i}
\end{array}\right)
$$

Applying (2.12) to $F$ and $E_{\left(R_{i},-P_{i}\right)}(s)$, we get $b_{i} \varepsilon=0$, i.e. $b_{i}=0$. This is true for all $i=1, \ldots, \sigma(A)$, therefore $F$ is cuspidal. Q.E.D.

THEOREM 5. For each $\lambda \neq \pm 2 \sqrt{q}$ we have

$$
\mathfrak{N}(\Gamma(A), \lambda)=\mathcal{E}(\Gamma(A), \lambda) \oplus \mathcal{C}(\Gamma(A), \lambda) .
$$

Proof. Write $\lambda=q^{s / 2}+q^{1-s / 2}$ for some $s$ with $\operatorname{Re} s>1$. Let

$$
\left(\begin{array}{c}
P_{i} \\
R_{i}
\end{array}\right), \quad i=1, \ldots, \sigma(A),
$$

be the cusps of $\Gamma(A)$ and, say, $\left(\begin{array}{c}P_{R_{1}} \\ )\end{array}\right)=\left(\begin{array}{l}A \\ 1\end{array}\right)$.

Case I. $\lambda \neq \pm 2 \sqrt{q}, \pm(q+1)$. Given $F$ in $\mathscr{N}(\Gamma(A), \lambda)$, there exist $\alpha_{i}$, 
$i=1, \ldots, \sigma(A)$, in $\mathrm{C}$ such that $G=F-\sum_{i=1}^{\sigma(A)} \alpha_{i} E_{\left(P_{i}, R_{i}\right)}(s)$ has zero $q^{n s / 2}$ part on each cusp. Then $G$ is in $\mathcal{E}(\Gamma(A), \lambda)$ by Lemma 3 .

Case II. $\lambda= \pm(q+1)$, i.e., $s=2+2 n \pi i / \log q$ for some $n \in Z$ Z. Let $F \in$ $\Re(\Gamma(A), \lambda)$. From the proof of Theorem 2, Case II, one sees that there exist $\alpha_{i}, i=2, \ldots, \sigma(A)$, in $C$ such that $G=F-\sum_{i=2}^{\sigma(A)} \alpha_{i} \tilde{E}_{\left(P_{i}, R_{)}\right)}(s)$ has zero $q^{n s / 2}$ part on each cusp $\left(P_{R_{i}}\right) \neq\left({ }_{A}^{1}\right)$. We claim that $G$ has zero $q^{n s} / 2$ part on the cusp $\left({ }_{A}^{1}\right)$ too. If this is so, then applying $(2.12)$ to $G$ and $\tilde{E}_{\left(P_{i}, R_{i}\right)}(s)$, we get

$$
b_{\left(-R_{i}, P_{i}\right)} \varepsilon-b_{\left(-R_{1}, P_{1}\right)} \varepsilon=0 \text {, }
$$

i.e.,

$$
b_{\left(-R_{i}, P_{i}\right)}=b_{\left(-R_{1}, P_{1}\right)}
$$

for all $i=2, \ldots, \sigma(A)$, where $b_{\left(-R_{,}, P_{j}\right)}$ is the $q^{n(1-s / 2)}$ part of $G$ on the cusp $\left(\bar{P}_{j} R_{j}\right), j=1, \ldots, \sigma(A)$. This shows that $G$ has the same $q^{n(1-s / 2)}$ part on each cusp. After subtracting a suitable constant function or alternating constant function from $G$, according as $\lambda=q+1$ or $-(q+1)$, we have the resulting function in $\mathcal{C}(\Gamma(A), \lambda)$. We now prove the claim. Suppose to the contrary, $G$ has nonzero $q^{n s / 2}$ part on the cusp $\left({ }_{A}^{1}\right)$. Let $\gamma_{1}, \ldots, \gamma_{n}$ be left coset representatives for $\Gamma(A)$ in $\Gamma$; then $\tilde{G}=\sum_{i=1}^{n} \gamma_{i} \cdot G$ is a $\lambda$-harmonic modular function for $\Gamma$. There is only one cusp in the fundamental domain $\mathscr{D}(1)$ and, by assumption, $\tilde{G}$ has nonzero $q^{n s} / 2$ part on that cusp. This is impossible as we will see from the following:

LEMMA 4. Let $F$ be a nonzero $\lambda$-harmonic modular function for $\Gamma$ with $\lambda=q^{s / 2}+q^{1-s / 2}, \operatorname{Re} s \geqslant 1$. Write

$$
F\left(\left[\sigma_{n}\right]\right)= \begin{cases}a \cdot q^{n s / 2}+b \cdot q^{n(1-s / 2)} & \text { if } \lambda \neq \pm 2 \sqrt{q}, \\ a \cdot q^{n s / 2}+b \cdot n \cdot q^{n(1-s / 2)} & \text { if } \lambda= \pm 2 \sqrt{q},\end{cases}
$$

for all $n>0$. Then $a \cdot b \neq 0$ if $\lambda \neq \pm(q+1) ; a=0$ if $\lambda= \pm(q+1)$. Moreover, $\operatorname{dim} \mathfrak{T}(\Gamma, \lambda)=1$ for all $\lambda$.

Proof. Note that $\lambda F\left(\left[\sigma_{0}\right]\right)=(q+1) F\left(\left[\sigma_{1}\right]\right)$.

Case I. $\lambda= \pm 2 \sqrt{q}$. Then

$$
\lambda a=\left(q^{s / 2}+q^{1-s / 2}\right) \cdot a=(q+1)\left(a q^{s / 2}+b q^{1-s / 2}\right),
$$

i.e.,

$$
\left(q^{1-s / 2}-q^{1+s / 2}\right) a=(q+1) \cdot q^{1-s / 2} \cdot b,
$$

i.e.,

$$
\left(1-q^{s}\right) a=(q+1) b .
$$

This implies $a \cdot b \neq 0$ since $q^{s} \neq 1$ and $F \neq 0$.

Case II. $\lambda \neq \pm 2 \sqrt{q}$. Then

$$
\lambda(a+b)=\left(q^{s / 2}+q^{1-s / 2}\right)(a+b)=\left(a \cdot q^{s / 2}+b \cdot q^{1-s / 2}\right)(q+1),
$$


i.e.,

$$
\left(q^{1-s / 2}-q^{1+s / 2}\right) a=\left(q^{2-s / 2}-q^{s / 2}\right) b,
$$

i.e.,

$$
q\left(1-q^{s}\right) a=\left(q^{2}-q^{s}\right) b .
$$

Since $q^{s} \neq 1$ and $F \neq 0$, it follows immediately that $a \cdot b \neq 0$ if $q^{2} \neq q^{s}$, i.e., $\lambda \neq \pm(q+1)$; and $a=0$ if $q^{2}=q^{s}$, i.e., $\lambda= \pm(q+1)$. It is clear that as long as $\lambda \neq \pm(q+1)$, there is a nontrivial relation between $a$ and $b$, as shown in Cases I and II above, so $\mathfrak{T}(\Gamma, \lambda)$ is one dimensional for all $\lambda$. Q.E.D.

\section{Corollary 2. $\mathscr{T}(\Gamma(A), \lambda)=\mathcal{E}(\Gamma(A), \lambda)$ if $|\lambda| \geqslant q+1$.}

Proof. Apply Theorems 4 and 5. Q.E.D.

2.3. Functional equations. As observed before, for all but a discrete set of $s$ in $\mathrm{C}$, the functions $E_{(P, R)}(s)$ defined by (2.2) are $\lambda$-harmonic modular functions for $\Gamma(A)$ with $\lambda=q^{s / 2}+q^{1-s / 2}$. Since the $\lambda$ 's remain the same if we replace $s$ by $2-s$, the functions $E_{(P, R)}(2-s)$, with $\operatorname{Re} s \geqslant 1$, are in $\Re\left(\Gamma(A), q^{s / 2}+q^{1-s / 2}\right)$. In fact, they are in $\mathcal{E}\left(\Gamma(A), q^{s / 2}+q^{1-s / 2}\right)$. To see this, it suffices to show that $E_{(A, 1)}(2-s)$ is, for the others are obtained from $E_{(A, 1)}(2-s)$ by left translations by $\Gamma$ and $\mathcal{E}\left(\Gamma(A), q^{s / 2}+q^{1-s / 2}\right)$ is invariant under left translations by $\Gamma$.

First note that for $\operatorname{Re} s>1,\left(\bar{P}_{P}^{R}-S\right) \in \Gamma, E_{(A, 1)}(2-s)$ has $q^{n s / 2}$ part equal to $q^{1-a} \Phi_{(P,-Q)}(0,2-s)$ and $q^{n(1-s / 2)}$ part equal to $\delta_{P, A} \delta_{-Q, 1} \cdot \varepsilon$ on the cusp $\left({ }_{P}^{-R}\right)$. From now on let $\gamma_{i}=\left(\begin{array}{c}P_{i} \\ R_{i} S_{i}\end{array}\right), i=1, \ldots, \sigma(A)$, be fixed elements in $\Gamma$ which determine all cusps $\left(\begin{array}{c}P_{i} \\ R_{i}\end{array}\right)$ of $\Gamma(A)$ and, say, $\gamma_{1}=\left(\begin{array}{ll}1 & 1 \\ 0 & 1\end{array}\right)$. Since $E_{\left(P_{i}, R_{j}\right)}(s)$ is the only one among $E_{\left(P_{j}, R_{j}\right)}(s), j=1, \ldots, \sigma(A)$, which has nonzero $q^{n s}$ part $\varepsilon$ on the cusp $\left(\bar{P}_{i} R_{i}\right)$, we see that, for $\operatorname{Re} s>1$,

$$
E_{(A, 1)}(2-s)-\sum_{i=1}^{\sigma(A)} \frac{1}{\varepsilon} \cdot q^{1-a} \Phi_{\left(P_{i}, Q_{i}\right)}(0,2-s) E_{\left(P_{i}, R_{i}\right)}(s)
$$

has zero $q^{n s / 2}$ part on each cusp of $\Gamma(A)$. Hence, by Lemma 3, it is cuspidal. However, Corollary 2 shows that $(2.13)$ is identically zero on certain halfplane $\operatorname{Re} s>c$. The function (2.13), when evaluated at each element [ $g$ ] of $\mathfrak{B}$, is meromorphic on $\mathrm{C}$ with a discrete set of poles and vanishes on $\operatorname{Re} s>c$. Therefore it must be identically zero on the entire $s$-plane. This proves

$$
E_{(A, 1)}(2-s)=\sum_{i=1}^{\sigma(A)} \frac{1}{\varepsilon} \cdot q^{1-a} \cdot \Phi_{\left(P_{i},-Q_{i}\right)}(0,2-s) E_{\left(P_{i}, R_{i}\right)}(s)
$$

for all $s$. Moreover, (2.8) shows that $\Phi_{(P, R)}(0, s)$ is independent of $R$ as long as 
$(P, R, A)=1$, so we can replace $\Phi_{\left(P_{i},-Q\right)}$ in (2.14) by $\Phi_{\left(P_{i}, R_{i}\right)}$ and obtain

$$
E_{(A, 1)}(2-s)=\sum_{i=1}^{\sigma(A)} \frac{1}{\varepsilon} q^{1-a} \cdot \Phi_{(A, 1) \gamma_{i}^{-1}}(0,2-s) \cdot E_{(A, 1) \gamma_{i}^{-1}}(s) .
$$

Let $\gamma_{j}^{-1}$ act on (2.15). For $1<i<\sigma(A)$, there is a unique $m, 1<m<\sigma(A)$, depending on $i$ and $j$, such that $(A, 1) \gamma_{i}^{-1} \gamma_{j}^{-1} \equiv(A, 1) \gamma_{m}^{-1}$, i.e., $(A, 1) \gamma_{i}^{-1} \equiv$ $(A, 1) \gamma_{m}^{-1} \gamma_{j}$. Different $i$ 's give different $m$ 's. Therefore, the action of $\gamma_{j}^{-1}$ on (2.15) can be described by

$$
E_{(A, 1) \gamma_{j}^{-1}}(2-s)=\sum_{i=1}^{\sigma(A)} \frac{1}{\varepsilon} q^{1-a} \Phi_{(A, 1) \gamma_{i}^{-1} \gamma_{j}}(0,2-s) E_{(A, 1) \gamma_{i}^{-1}}(s) .
$$

We have proven

THEOREM 6. Let $\gamma_{i}, i=1, \ldots, \sigma(A)$, with $\gamma_{1}=\left(\begin{array}{ll}1 & 0 \\ 0 & 1\end{array}\right)$ be fixed elements in $\Gamma$, such that they determine all the cusps of $\Gamma(A)$. Then for $1<j<\sigma(A)$,

$$
E_{(A, 1) \gamma_{j}^{-1}}(2-s)=\sum_{i=1}^{\sigma(A)} \frac{1}{\varepsilon} q^{1-a} \Phi_{(A, 1) \gamma_{i}^{-1} \gamma_{j}}(0,2-s) E_{(A, 1) \gamma_{i}^{-1}}(s)
$$

holds on the entire s-plane.

The relations among $\Phi_{(P, R)}(0, s)$ are described by

TheOREM 7. Notation is as above. For $1<i, j<\sigma(A)$, put

$$
\Psi_{i j}(s)=(1 / \varepsilon) q^{1-a} \Phi_{(A, 1) \gamma_{i}^{-1} \gamma_{j}}(0, s)
$$

and form the $\sigma(A) \times \sigma(A)$ matrix $\Psi(s)=\left(\Psi_{i j}(s)\right)$. Then $\Psi(s)$ is symmetric and satisfies

$$
\Psi(2-s) \cdot \Psi(s)=I
$$

for all $s$, where $I$ is the $\sigma(A) \times \sigma(A)$ identity matrix.

Proof. Say

$$
\gamma_{i}^{-1} \gamma_{j}=\left(\begin{array}{ll}
P & Q \\
R & S
\end{array}\right)
$$

Then

$$
\gamma_{j}^{-1} \gamma_{i}=\left(\begin{array}{cc}
S & -Q \\
-R & P
\end{array}\right)
$$

and, by (2.8),

$$
\begin{aligned}
\Phi_{(A, 1) \gamma_{i}^{-1} \gamma_{j}}(0, s) & =\Phi_{(R, S)}(0, s)=\Phi_{(-R, P)}(0, s) \\
& =\Phi_{(A, 1) \gamma_{j}^{-1} \gamma_{i}}(0, s) .
\end{aligned}
$$

This shows that $\Psi_{i j}(s)=\Psi_{j i}(s)$, i.e., $\Psi(s)$ is symmetric. Now fix $j, 1<j<$ $\sigma(A)$, and look at the $q^{n(1-s / 2)}$ part $(\operatorname{Re} s>1)$ of $(2.16)$ on the cusp 
determined by $\gamma_{l}:$ If $l \neq j$, we get

$$
0=\sum_{i=1}^{\sigma(A)} \frac{1}{\varepsilon} q^{1-a} \Phi_{(A, 1) \gamma_{i}^{-1} \gamma_{j}}(0,2-s) \cdot q^{1-a} \cdot \Phi_{(A, 1) \gamma_{i}^{-1} \gamma_{i}}(s)
$$

i.e.,

$$
0=\sum_{i=1}^{\sigma(A)} \Psi_{i j}(2-s) \cdot \Psi_{i l}(s)
$$

and if $l=j$, we get

$$
\varepsilon=\sum_{i=1}^{\sigma(A)} \frac{1}{\varepsilon} q^{1-a} \Phi_{(A, 1) \gamma_{i}^{-1} \gamma_{j}}(0,2-s) \cdot q^{1-a} \Phi_{(A, 1) \gamma_{i}^{-1} \gamma_{j}}(s)
$$

i.e.,

$$
1=\sum_{i=1}^{\sigma(A)} \Psi_{i j}(2-s) \cdot \Psi_{i j}(s)
$$

We have proven that (2.17) holds for $\operatorname{Re} s>1$, hence it holds for all $s$ since each $\Psi_{i j}(s)$ is meromorphic on the whole $s$-plane with a discrete set of poles. Q.E.D.

REMARK. In matrix notation, Theorem 6 reads

$$
\begin{aligned}
\left(E_{(A, 1) \gamma_{1}^{-1}}(s)\right. & \left.\ldots, E_{(A, 1) \gamma_{o}(A)}(s)\right) \cdot \Psi(2-s) \\
= & \left(E_{(A, 1) \gamma_{1}^{-1}}(2-s), \ldots, E_{\left.(A, 1) \gamma_{o}-1\right)}(2-s)\right) .
\end{aligned}
$$

3. Eisenstein series and decomposition theory (Part II: $\lambda= \pm 2 \sqrt{q}$ ).

3.1. The space $\mathcal{E}(\Gamma(A), \pm 2 \sqrt{q})$. Notation is as in $\S 2.3$. When $s=1+$ $2 n \pi i / \log q, n \in \mathbf{Z},(2.18)$ gives

$$
\begin{aligned}
\left(E_{(A, 1) \gamma_{1}^{-1}}(s),\right. & \left.\ldots, E_{(A, 1) \gamma_{o}(A)}(s)\right) \cdot \Psi(s) \\
& =\left(E_{(A, 1) \gamma_{1}^{-1}}(s), \ldots, E_{(A, 1) \gamma_{o}-A}(s)\right)
\end{aligned}
$$

and (2.17) becomes $\Psi^{2}(s)=\Psi^{2}(1)=I$ since $\Psi(s)$ has period $2 \pi i / \log q$. Let $B$ be a $\sigma(A) \times \sigma(A)$ matrix such that $B^{-1} \cdot \Psi(1) \cdot B$ is diagonal. Simple computation shows that

$$
\Psi_{i i}(1)=\frac{1}{\varepsilon} q^{1-a} \Phi_{(A, 1)}(0,1)=-\frac{q-1}{\varphi(A)} \cdot \frac{1}{\varepsilon} \neq 1
$$

for all $i=1, \ldots, \sigma(A)$. This, in particular, implies that $\Psi(1)$ is not the 
identity matrix so we may assume that $B$ is chosen so that

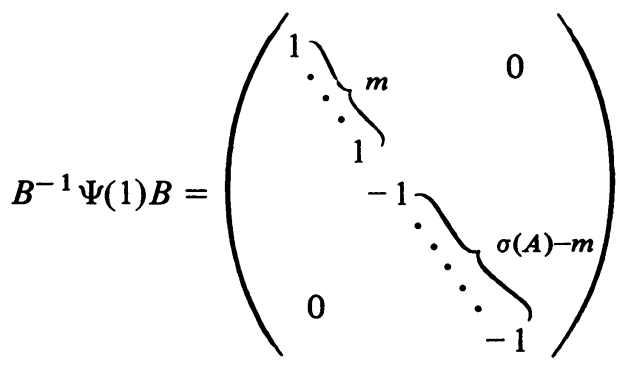

with $\sigma(A)>m>0$. Thus, as $s=1+2 n \pi i / \log q, n \in \mathbf{Z}$, (3.1) reads

$$
\left(E_{(A, 1) \gamma_{1}^{-1}}(s), \ldots, E_{(A, 1) \gamma_{0}(\hat{A})}(s)\right) B \cdot B^{-1} \cdot \Psi(1) \cdot B
$$

$$
\begin{aligned}
& =\left(E_{(A, 1) \gamma_{1}^{-1}}(s), \ldots, E_{(A, 1) \gamma_{0}(\lambda)}(s)\right) \cdot B \cdot(\underbrace{1}_{-1} \cdot E_{(A, 1) \gamma_{o}(\lambda)}(s)) \cdot B . \\
& =\left(E_{(A, 1) \gamma_{1}^{-1}}(s), \ldots(A)-m\right) \\
& 0
\end{aligned}
$$

Setting

$$
\left(E_{(A, 1) \gamma_{1}^{-1}}(s), \ldots, E_{(A, 1) \gamma_{o}(A)}(s)\right) \cdot B=\left(G_{1}(s), \ldots, G_{\sigma(A)}(s)\right),
$$

(3.3) shows that $G_{m+1}(s)=\cdots=G_{o(A)}(s)=0$. Therefore, when $s=1+$ $2 n \pi i / \log q, n \in \mathbf{Z}$, the space generated by $E_{(A, 1) \gamma_{i}{ }^{-1}}(s), i=1, \ldots, \sigma(A)$, which is the same as that generated by all $E_{(P, R)}(s)$, is in fact generated by $G_{1}(s), \ldots, G_{m}(s)$ and has dimension at most equal to $m$, which is less than $\sigma(A)$. In particular, when $A$ is a constant, the $E_{(P, R)}(s)$ 's are all zero.

Here, the situation is different from that in the classical case. In the classical theory, as developed in Hecke [6, No. 24], Eisenstein series of weight 1 for the principal congruence subgroup $\Gamma(N)$ are obtained from analytic continuation; and they generate a space of dimension equal to half the number of cusps of $\Gamma(N)$ (if $N>2$ ). Moreover, the space of modular forms of weight 1 for $\Gamma(N)$ is generated by these Eisenstein series and by cusp forms. However, in the present case, the vector space complement of $\mathcal{C}(\Gamma(A), \pm 2 \sqrt{q})$ in $\Re(\Gamma(A), \pm 2 \sqrt{q})$ has dimension greater than that of the space generated by the Eisenstein series obtained from analytic continuation. In other words, there are some functions, linearly independent of $E_{\left(P_{i}, R_{i}\right)}(s), i=1, \ldots, \sigma(A), s=1+n \pi i / \log q$, which should also be called "Eisenstein series". 
To discover these "missing" $\lambda$-harmonic Eisenstein series for $\Gamma(A)$ with $\lambda= \pm 2 \sqrt{q}$, we consider the derivative of each $E_{(P, R)}([g])(s)$ with respect to $s$, denoted by $E_{(P, R)}^{\prime}([g])(s)$.

TheOReM 8. Let $\left[\left(\begin{array}{c}T^{n} \\ 0\end{array}\right)\right] \in \mathfrak{S}, P, R \in \mathbf{F}_{q}[T]$ with $(P, R, A)=1$. Then the function $E_{(P, R)}\left(\left[\left(T_{0}^{n} y_{1}^{n}\right)\right], s\right)$, viewed as a function of $s$, is differentiable at $s=1+$ $2 m \pi i / \log q, \quad m \in \mathbf{Z}$, and its derivative, $E_{(P, R)}^{\prime}\left(\left[\left(C_{0}^{T^{n}} \begin{array}{l}y \\ 1\end{array}\right)\right], s\right)$ at $s=1+$ $2 m \pi i / \log q, m \in \mathbf{Z}$, when viewed as a function on $\mathfrak{B}$, is a $\lambda$-harmonic modular function for $\Gamma(A)$ with $\lambda=q^{s / 2}+q^{1-s / 2}$.

Proof. $E_{(P, R)}\left(\left[\left(\begin{array}{c}T^{n} \\ 0\end{array}\right)\right]\right)$ is differentiable at $s=1+2 m \pi i / \log q, m \in \mathbf{Z}$, by Theorem 1. Since for all $\gamma_{A} \in \Gamma(A),[g] \in \mathscr{G}_{(P, R)}\left(\left[\gamma_{A} g\right]\right)(s)=E_{(P, R)}([g])(s)$ for all $s, E_{(P, R)}^{\prime}\left(\left[\gamma_{A} g\right]\right)(s)=E_{(P, R)}^{\prime}([g])(s)$ as long as the derivative exists. This shows, in particular, that at $s=1+2 m \pi i / \log q, m \in \mathbf{Z}, E_{(P, R)}^{\prime}(s)$ is a modular function for $\Gamma(A)$. Finally, to show $\lambda$-harmonicity of $E_{(P, R)}^{\prime}$ at $s=1+$ $2 m \pi i / \log q, m \in \mathbf{Z}$, one looks at the Fourier coefficients $C_{(P, R)}^{\prime}(n, Q, s)$ of $E_{(P, R)}^{\prime}(s)$ at $s=1+2 m \pi i / \log q, m \in \mathbf{Z}$. They are

$$
\begin{aligned}
C_{(P, R)}^{\prime}(n, 0, s)= & \frac{\log q}{2}\left(\delta_{P, A} \delta_{R, 1} \varepsilon-q^{1-a} \Phi_{(P, R)}(0, s)\right) n q^{n \cdot s / 2} \\
& +q^{1-a} \cdot \Phi_{(P, R)}^{\prime}(0, s) q^{n \cdot s / 2}
\end{aligned}
$$

and for $Q \neq 0, d \geqslant 0$,

$$
\begin{aligned}
& C_{(P, R)}^{\prime}(a-2-\text { ord } Q-d, Q, s)=-\frac{\log q}{2} \Phi_{(P, R)}(Q, s) \cdot q^{1-a} \\
& \cdot \sum_{m=0}^{d} q^{m(1-s)}(a-2-\operatorname{ord} Q-d) \cdot q^{(a-2-\operatorname{ord} Q-d) \cdot s / 2} \\
& +q^{1-a}\left[\Phi_{(P, R)}^{\prime}(Q, s) \cdot \sum_{m=0}^{d} q^{m(1-s)}-\Phi_{(P, R)}(Q, s) \cdot \sum_{m=0}^{d} m \cdot q^{m(1-s)} \log q\right] \\
& \quad \cdot q^{(a-2-\operatorname{ord} Q-d) \cdot s / 2} .
\end{aligned}
$$

In particular, the Fourier coefficients are of the form $a \cdot n \cdot q^{n s / 2}+b \cdot q^{n s / 2}$, and hence satisfy (1.2) with $\lambda=q^{s / 2}+q^{1-s / 2}$. Q.E.D.

For $s=1+2 n \pi i / \log q, n \in \mathbf{Z}$, let $\mathcal{E}\left(\Gamma(A), q^{s / 2}+q^{1-s / 2}\right)$, the space of $\lambda$-harmonic Eisenstein series for $\Gamma(A)$ with $\lambda=q^{s / 2}+q^{1-s / 2}$, be the space generated by all $E_{(P, R)}(s)$ and all $E_{(P, R)}^{\prime}(s)$. We shall see that the dimension of $\mathcal{E}(\Gamma(A), \lambda)$ is $\sigma(A)$, and that the space $\Re(\Gamma(A), \lambda)$ is the direct sum of $\mathcal{E}(\Gamma(A), \lambda)$ and $\mathcal{C}(\Gamma(A), \lambda)$. In other words, by considering $E_{(P, R)}^{\prime}(s)$ we have obtained all the "missing" Eisenstein series.

3.2. Decomposition theory of $\mathscr{N}(\Gamma(A), \pm 2 \sqrt{q})$. Throughout this section, $s$ is equal to $1+2 l \pi i / \log q$ for some $l \in \mathbf{Z}$, therefore 
$\lambda=q^{s / 2}+q^{1-s / 2}=2 \sqrt{q}$ or $-2 \sqrt{q}$ according as $l$ is even or odd. Let $\gamma_{i} \in \Gamma, i=1, \ldots, \sigma(A)$, be as in \$3.1, namely, $\gamma_{1}=\left(\begin{array}{ll}1 & 9 \\ 0 & 1\end{array}\right)$ and the $\gamma_{i}^{\prime}$ 's determine all the cusps of $\Gamma(A)$.

THEOREM 9. The space $\mathcal{E}\left(\Gamma(A), q^{s / 2}+q^{1-s / 2}\right)$ has dimension equal to $\sigma(A)$, the number of cusps of $\Gamma(A)$.

Proof. We have shown in $\$ 3.1$ that the space generated by all $E_{(A, 1) y_{i}^{-1}}(s)$, $i=1, \ldots, \sigma(A)$, is in fact generated by $G_{1}(s), \ldots, G_{m}(s)$ where

$$
\left(E_{(A, 1) \gamma_{1}^{-1}}(s), \ldots, E_{(A, 1) \gamma_{o}(\bar{A})}(s)\right) \cdot B=\left(G_{1}(s), \ldots, G_{m}(s), 0, \ldots, 0\right) .
$$

We first prove that $G_{1}(s), \ldots, G_{m}(s)$ are linearly independent. Note that when $s=1+2 l \pi i / \log q$, the value of $E_{(A, 1) \gamma_{i}^{-1}}(s)$ on the cusp determined by $\gamma_{j}$ is

$$
\left(\delta_{i j} \varepsilon+q^{1-a} \Phi_{(A, 1) \gamma_{i}^{-1} \gamma_{j}}(0, s)\right) \cdot q^{n s / 2}=\varepsilon\left(\delta_{i j}+\Psi_{i j}(1)\right) q^{n s / 2} \text { for } n \geqslant a-1,
$$

where $\delta_{i j}$ is the Kronecker delta function. We say that $(I+\Psi(1)) \cdot \varepsilon$ is the matrix associated to the $q^{n s / 2}$ part of

$$
\left(E_{(A, 1) \gamma_{1}^{-1}}(s), \ldots, E_{(A, 1) \gamma_{o}(\bar{A})}(s)\right) .
$$

Therefore the matrix associated to $1 / \varepsilon$ times the $q^{n s / 2}$ part of $\left(G_{1}(s), \ldots, G_{m}(s), 0, \ldots, 0\right)$ is

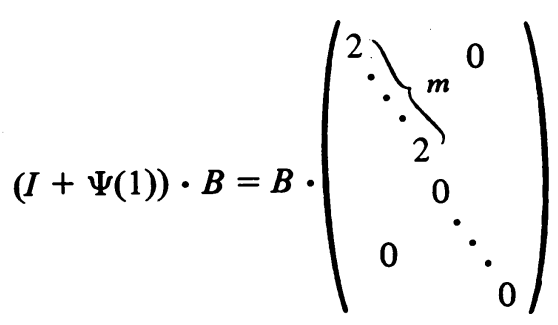

by (3.2). This implies that $G_{1}(s), \ldots, G_{m}(s)$ are linearly independent. Now look at $E_{(A, 1) \gamma_{i}^{-1}}^{\prime}(s)$. Put

$$
\left(E_{(A, 1) \gamma_{1}^{-1}}^{\prime}(s), \ldots, E_{(A, 1) \gamma_{o}(A)}^{\prime}(s)\right) \cdot B=\left(\tilde{G}_{1}(s), \ldots, \tilde{G}_{\sigma(A)}(s)\right) .
$$

(3.5) shows that the matrix associated to the $n \cdot q^{n s / 2}$ part of

$$
\left(E_{(A, 1) \gamma_{1}^{-1}}^{\prime}(s), \ldots, E_{(A, 1) \gamma_{o}(-1)}^{\prime}(s)\right)
$$

is $(\varepsilon / 2) \log q$ times $I-\Psi(1)$, therefore the matrix associated to the $n \cdot q^{n s / 2}$ 
part of $\left(\tilde{G}_{1}(s), \ldots, \tilde{G}_{\sigma(A)}(s)\right)$ is $(\varepsilon / 2) \log q$ times

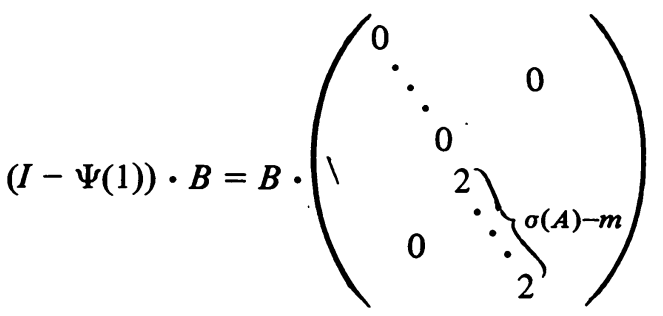

This implies the linear independence of $\tilde{G}_{m+1}(s), \ldots, \tilde{G}_{o(A)}(s)$. It is obvious that $G_{1}(s), \ldots, G_{m}(s), \tilde{G}_{m+1}(s), \ldots, \tilde{G}_{o(A)}(s)$ are linearly independent since $G_{1}(s), \ldots, G_{m}(s)$ have zero $n q^{n \cdot s / 2}$ part on each cusp. Finally, we prove that $\mathcal{E}\left(\Gamma(A), q^{s / 2}+q^{1-s / 2}\right)$ is generated by $G_{1}(s), \ldots, G_{m}(A)$, $\tilde{G}_{m+1}(s), \ldots, \tilde{G}_{\sigma(A)}(s)$. Evaluating $(2.18)$ at $[g] \in \mathscr{S}$ and differentiating it at $s=1+2 l \pi i / \log q$, we obtain

$$
\begin{aligned}
\left(E_{(A, 1) \gamma_{1}^{-1}}^{\prime}([g], s),\right. & \left.\ldots, E_{(A, 1) \gamma_{o}^{-1} A}^{\prime}([g], s)\right) \cdot \Psi(2-s) \\
& -\left(E_{(A, 1) \gamma_{1}^{-1}}([g], s), \ldots, E_{(A, 1) \gamma_{o}(A)}([g], s)\right) \cdot \Psi^{\prime}(2-s) \\
& =-\left(E_{(A, 1) \gamma_{1}^{-1}}^{\prime}([g], 2-s), \ldots, E_{(A, 1) \gamma_{o}(A)}^{\prime}([g], 2-s)\right)
\end{aligned}
$$

where $\Psi^{\prime}(s)$ is the matrix whose $i j$ th term is $\Psi_{i j}^{\prime}(s)$. In other words, we have

$$
\begin{aligned}
\left(E_{(A, 1) \gamma_{1}^{-1}}^{\prime}(s)\right. & \left.\ldots, E_{(A, 1) \gamma_{o}(A)}^{\prime}(s)\right) \cdot(I+\Psi(1)) \\
& =\left(E_{(A, 1) \gamma_{1}^{-1}}(s), \ldots, E_{(A, 1) \gamma_{o}(A)}(s)\right) \cdot \Psi^{\prime}(2-s)
\end{aligned}
$$

or, equivalently,

$$
\begin{aligned}
\left(E_{(A, 1) \gamma_{1}^{-1}}^{\prime}(s), \ldots, E_{(A, 1) \gamma_{0}(\lambda)}^{\prime}(s)\right) \cdot B \cdot B^{-1} \cdot(I+\Psi(1)) \cdot B \\
\quad=\left(E_{(A, 1) \gamma_{1}^{-1}}(s), \ldots, E_{(A, 1) \gamma_{0}^{-}(\hat{A})}(s)\right) \cdot B \cdot B^{-1} \cdot \Psi^{\prime}(2-s) \cdot B
\end{aligned}
$$

i.e.,

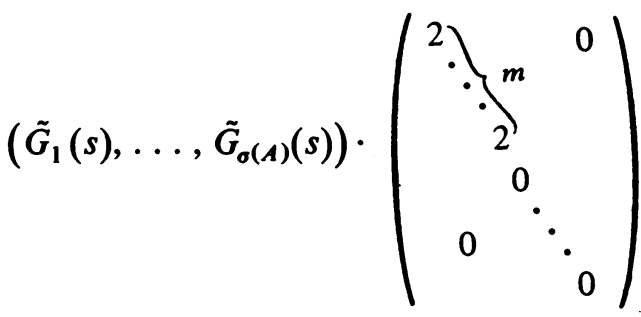

$$
\begin{aligned}
& =\left(G_{1}(s), \ldots, G_{m}(s), 0, \ldots, 0\right) \cdot B^{-1} \cdot \Psi^{\prime}(2-s) \cdot B .
\end{aligned}
$$

This means that $\tilde{G}_{1}(s), \ldots, \tilde{G}_{m}(s)$ are linear combinations of $G_{1}(s), \ldots, G_{m}(s)$; hence we have shown that $\mathcal{E}\left(\Gamma(A), q^{s / 2}+q^{1-s / 2}\right)$ is 
generated by $G_{1}(s), \ldots, G_{m}(s), \tilde{G}_{m+1}(s), \ldots, \tilde{G}_{\sigma(A)}(s)$, and thus is of dimension $\sigma(A)$. Q.E.D.

Now, we prove the decomposition theorem:

TheOREM 10. $\mathscr{T}(\Gamma(A), \lambda)=\mathcal{E}(\Gamma(A), \lambda) \oplus \mathcal{C}(\Gamma(A), \lambda), \lambda= \pm 2 \sqrt{q}$.

Proof. Write $\lambda=2 \cdot q^{s / 2}$ with $s=1+2 n \pi i / \log q$ for some $n \in Z$ Z. Let $F \in \Re\left(\Gamma(A), 2 \cdot q^{s / 2}\right)$. Assume that on the cusps determined by $\gamma_{i}, i=$ $1, \ldots, \sigma(A)$,

$$
F\left(\left[\gamma_{i} \sigma_{n}\right]\right)=a_{i}^{\prime} q^{n \cdot s / 2}+b_{i} n \cdot q^{n s / 2}
$$

for all $n \geqslant a-1$. Applying Corollary 2 to $F$ and $E_{(A, 1) \gamma_{i}^{-1}}(s), 1<i<\sigma(A)$, we get

$$
b_{i} \varepsilon+\sum_{j=1}^{\sigma(A)} b_{j} \cdot q^{1-a} \Phi_{(A, 1) \gamma_{i}^{-1} \gamma_{j}}(0, s)=0
$$

i.e.,

$$
b_{i}+\sum_{j=1}^{\sigma(A)} b_{j} \Psi_{i j}(1)=0
$$

for all $i=1, \ldots, \sigma(A)$, i.e.,

$$
\left(b_{1}, \ldots, b_{\sigma(A)}\right) \cdot(I+\Psi(1))=0 .
$$

If we view $\Psi(1)$ as a linear transformation on the $\sigma(A)$-dimensional space of row vectors, (3.2) shows clearly that the image of $I+\Psi(1)$ is the kernel of $I-\Psi(1)$, and the kernel of $I+\Psi(1)$ is the image of $I-\Psi(1)$. Therefore, there exist $\beta_{1}, \ldots, \beta_{\sigma(A)}$ in $C$ such that

$$
\left(b_{1}, \ldots, b_{\sigma(A)}\right)=\left(\beta_{1}, \ldots, \beta_{\sigma(A)}\right) \cdot(I-\Psi(1)) .
$$

However, $(\varepsilon / 2) \log q \cdot(I-\Psi(1))$ is the matrix associated to the $n \cdot q^{n \cdot s / 2}$ part of $\left(E_{(A, 1) \gamma_{1}^{-1}}^{\prime}(s), \ldots, E_{(A, 1) \gamma_{o}(A)}^{\prime-1}(s)\right)$; this implies

$$
G=F-2 \cdot \frac{1}{\varepsilon} \cdot \frac{1}{\log q} \cdot \sum_{i=1}^{\sigma(A)} \beta_{i} E_{(A, 1) \gamma_{i}^{-1}}^{\prime}(s)
$$

has zero $n \cdot q^{n s / 2}$ part on each cusp. Write

$$
G\left(\left[\gamma_{i} \sigma_{n}\right]\right)=a_{i} q^{n \cdot s / 2} \text { for } i=1, \ldots, \sigma(A), \quad n \geqslant a-1 .
$$

Applying Corollary 1 to $G$ and $E_{(A, 1) \gamma_{i}^{-1}}^{\prime}(s)$ for $i=1, \ldots, \sigma(A)$, we get

$$
\left(a_{1}, \ldots, a_{\sigma(A)}\right) \cdot(I-\Psi(1))=0 \text {. }
$$

Therefore there exist $\alpha_{1}, \ldots, \alpha_{\sigma(A)}$ in $\mathbf{C}$ such that

$$
\left(a_{1}, \ldots, a_{\sigma(A)}\right)=\left(\alpha_{1}, \ldots, \alpha_{\sigma(A)}\right)(I+\Psi(1)) .
$$

Note that the matrix associated to the $q^{n s / 2}$ part of $\left(E_{(A, 1) \gamma_{1}^{-1}}(s), \ldots\right.$, 
$E_{\left.(A, 1) \gamma_{\sigma(\lambda)}^{-1}(s)\right)}($ is $\varepsilon \cdot(I+\Psi(1))$. So

$$
G-\frac{1}{\varepsilon} \cdot \sum_{i=1}^{\sigma(A)} \alpha_{i} \cdot E_{(A, 1) \gamma_{i}^{-1}}(s)
$$

has zero $q^{n s / 2}$ part as well as zero $n \cdot q^{n s / 2}$ part on each cusp, i.e., is cuspidal. This completes the proof of the theorem. Q.E.D.

4. Examples of cusp functions. For a monic polynomial $A$ in $\mathbf{F}_{q}[T]$, one may define

$$
\begin{aligned}
& \Gamma_{1}(A)=\left\{\left(\begin{array}{ll}
P & Q \\
R & S
\end{array}\right) \in \Gamma \mid P \equiv S \equiv 1 \bmod A, R \equiv 0 \bmod A\right\} \\
& \Gamma_{0}(A)=\left\{\left(\begin{array}{ll}
P & Q \\
R & S
\end{array}\right) \in \Gamma \mid R \equiv 0 \bmod A\right\}
\end{aligned}
$$

and form the space of cusp functions $C\left(\Gamma_{1}(A)\right), C\left(\Gamma_{0}(A)\right)$ for $\Gamma_{1}(A), \Gamma_{0}(A)$, respectively, as in the classical case. However, the dimensions of $\mathcal{C}(\Gamma(A))$, $\mathcal{C}\left(\Gamma_{1}(A)\right)$, and $\mathcal{C}(\Gamma(A))$ are still unknown. No systematic way of computing these dimensions is known to the author. Listed below are some examples of $\Gamma(A)$ and $\Gamma_{0}(A)$ for which the fundamental domains $\mathscr{D}(A), \mathscr{D}_{0}(A)$ and the spaces $\mathrm{C}(\Gamma(A)), \mathrm{C}\left(\Gamma_{0}(A)\right)$ are computed when $q=2$. We shall use $\sigma(A), \sigma_{0}(A)$ to denote the number of cusps of $\Gamma(A), \Gamma_{0}(A)$, respectively.

EXAMPLE 1. $A=T \cdot \sigma_{0}(T)=2$. $C\left(\Gamma_{0}(T)\right)=0$.

EXAMPLE 2. $A=T^{2} \cdot \sigma_{0}\left(T^{2}\right)=3 \cdot C\left(\Gamma_{0}\left(T^{2}\right)\right)=0$.

EXAmple 3. $A=T^{3} \cdot \sigma_{0}\left(T^{3}\right)=4$. $C\left(\Gamma_{0}\left(T^{3}\right)\right)=0$.

EXAmple 4. $A=T^{4} \cdot \sigma_{0}\left(T^{4}\right)=6, C\left(\Gamma_{0}\left(T^{4}\right)\right)=0$.

EXAmple 5. $A=T \cdot \sigma(T)=3$. $C(\Gamma(T))=0$.

EXAmple 6. $A=T^{2} \cdot \sigma\left(T^{2}\right)=12 . \bigodot\left(\Gamma\left(T^{2}\right)\right)=\bigodot\left(\Gamma\left(T^{2}\right), 0\right), 1$-dimensional.

The fundamental domains of these six groups are as follows:
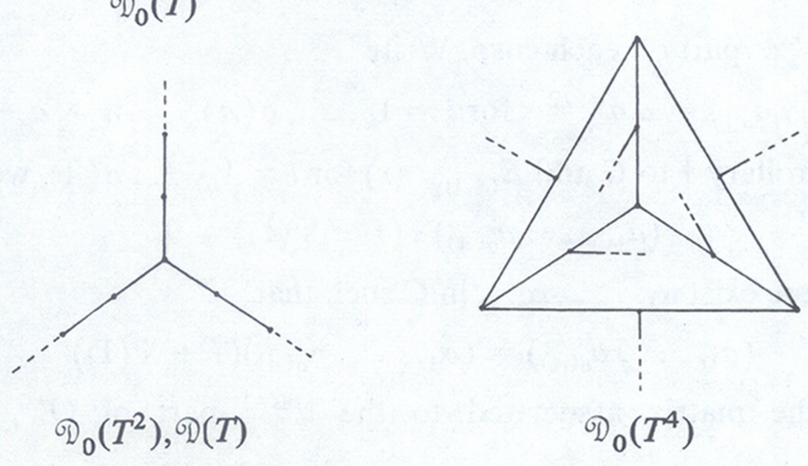

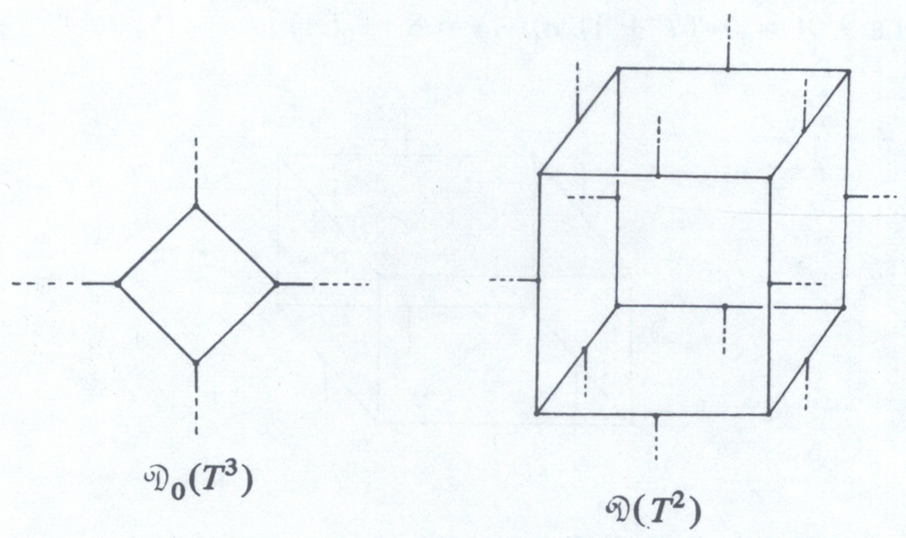

REMARK. The above six examples were computed by J. Weisinger.

EXAMPLE 7. $A=T^{3} \cdot \sigma\left(T^{3}\right)=48$.

$$
\begin{aligned}
& \mathcal{C}\left(\Gamma\left(T^{3}\right)\right)=\mathcal{C}\left(\Gamma\left(T^{3}\right), 0\right) \oplus \mathcal{C}\left(\Gamma\left(T^{3}\right),-\sqrt{2}\right) \\
& \quad \oplus \bigodot\left(\Gamma\left(T^{3}\right), \sqrt{2}\right) \oplus \bigodot\left(\Gamma\left(T^{3}\right),-2\right) \\
& \quad \oplus \bigodot\left(\Gamma\left(T^{3}\right), 2\right) \oplus \bigodot\left(\Gamma\left(T^{3}\right),-\sqrt{3+\sqrt{3}}\right) \oplus \bigodot\left(\Gamma\left(T^{3}\right), \sqrt{3+\sqrt{3}}\right) \\
& \quad \oplus \bigodot\left(\Gamma\left(T^{3}\right),-\sqrt{3-\sqrt{3}}\right) \oplus \bigodot\left(\Gamma\left(T^{3}\right), \sqrt{3-\sqrt{3}}\right),
\end{aligned}
$$

of dimensions $14,6,6,3,3,4,4,4,4$, respectively.

We remark here that $\operatorname{dim} \Re(G, \lambda)=\operatorname{dim} \Re(G,-\lambda)$ and $\operatorname{dim} C(G, \lambda)=$ $\operatorname{dim} \mathcal{C}(G,-\lambda)$ for any modular subgroup $G$ of $\operatorname{GL}\left(2, \mathbf{F}_{q}[T]\right)$ and for all $\lambda$ because of the following reason. Let $F \in \Re(G, \lambda)$. Define a new function $\tilde{F}$ on $\mathfrak{S}$ by

$$
\tilde{F}([g])= \begin{cases}F([g]) & \text { if } \operatorname{deg}[g] \text { is odd } \\ -F([g]) & \text { if } \operatorname{deg}[g] \text { is even }\end{cases}
$$

Then one sees that $\tilde{F} \in \Re(G,-\lambda)$. Moreover, $\tilde{F}$ is cuspidal if $F$ is. This gives a one-one correspondence between $\Re(G, \lambda)$ and $\Re(G,-\lambda)$, and also between $C(G, \lambda)$ and $C(G,-\lambda)$.

EXAMPLE 8. $A=\left(T^{3}+T+1\right)^{2} . \sigma_{0}(A)=9 . C\left(\Gamma_{0}(A)\right)=\bigodot\left(\Gamma_{0}(A), 0\right), 1-$ dimensional.

The fundamental domains for Examples 7 and 8 are too complicated, hence are omitted here. More interesting is the following 
ExAmple 9. $A=T^{3}(T+1), \sigma_{0}(A)=8 . \mathscr{D}_{0}(A)$ is

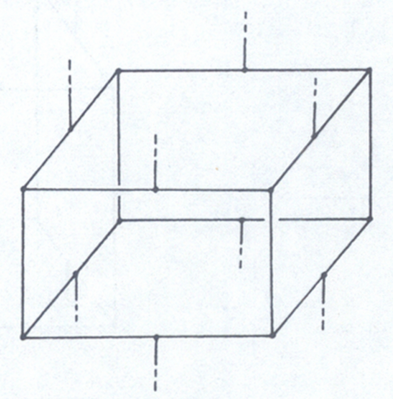

$\mathcal{C}\left(\Gamma_{0}(A)\right)=\mathcal{C}\left(\Gamma_{0}(A), 1\right) \oplus \mathcal{C}\left(\Gamma_{0}(A),-1\right)$, each of dimension one. J. Tate defined an elliptic curve by

$$
y^{2}+x y=x^{3}+1+1 / T,
$$

which has good reduction at the place $\infty$, and whose minimal model over $\mathbf{F}_{2}[T]$ is

$$
y^{2}+T x y=x^{3}+T^{6}+T^{5}
$$

with $\Delta=T^{11}(T+1), j=1 /(T+1)$, and conductor $=T^{3}(T+1)=A$. As a consequence of a more general result of Deligne [3], the zeta function of this elliptic curve gives rise to a cusp function $F$ for $\Gamma_{0}(A)$. Checking the Euler factor of this zeta function at the place $\infty$, one finds that $F$ lies in $\mathrm{C}\left(\Gamma_{0}(A),-\right.$ $1)$, and hence generates $\mathrm{C}\left(\Gamma_{0}(A),-1\right)$.

5. Concluding remarks. We have seen (in Theorems 5 and 10) that for all $\lambda$, the space $\Re(\Gamma(A), \lambda)$ of $\lambda$-harmonic modular functions for $\Gamma(A)$ is a direct sum of the space $\mathcal{E}(\Gamma(A), \lambda)$ of Eisenstein series, and the space $\mathcal{C}(\Gamma(A), \lambda)$ of cusp functions. Also, we have discussed $\mathcal{E}(\Gamma(A), \lambda)$ in detail, so what remains is the study of $\mathcal{C}(\Gamma(A), \lambda)$. For a function $F$ on $\mathfrak{g}$ and $M \in \mathrm{GL}(2, k)$. Let

$$
F \mid M([g])=F([M \cdot g]) \text { for all }[g] \in \mathfrak{B} \text {. }
$$

Then we can define "new functions" in $\mathcal{C}(\Gamma(A))$ just as we did in the classical case (cf. [9]), and the whole classical theory applies with suitable modifications.

It is interesting to compare the results in this paper with those on "nonanalytic" modular forms discussed in Maass [10, Chapter IV].

In $\S 4$, Example 9, we discussed a cusp function for $\Gamma_{0}\left(T^{3}(T+1)\right)$ coming from an elliptic curve. In general, it would be interesting to know how cusp functions for $\Gamma(A), \Gamma_{1}(A)$, and $\Gamma_{0}(A)$ arise; for example, is there any representation-theoretic explanation as in the classical case (cf. [2], [4])? Is there a moduli-theoretic interpretation for $\mathcal{C}(\Gamma(A)), \mathcal{C}\left(\Gamma_{1}(A)\right)$, and $\mathcal{C}\left(\Gamma_{0}(A)\right)$ ?

If one takes $k$ to be a function field in one variable over a finite field, and 
considers its completion $k_{v}$ at any place $v$, one may likewise study the modular functions defined on $\operatorname{GL}\left(2, k_{v}\right)$. One would like to know the decomposition theory in this situation.

\section{REFERENCES}

1. P. Cartier, Fonctions harmoniques sur un arbre, Ist. Naz. Alta Mat., Symposia Mathematica, 9 (1972), 203-270.

2. P. Deligne, Formes modulaires et représentations l-adiques, Séminaire Bourbaki, 1968/1969, Exp. 355, Lecture Notes in Math., vol. 179, Springer-Verlag, Berlin, 1971, pp. 139-172.

3. Les constantes des équations fonctionnelles des fonctions $L$, Modular Functions of One Variable. II, Lecture Notes in Math., vol. 349, Springer-Verlag, Berlin and New York, 1973, pp. 501-597.

4, P. Deligne and J.-P. Serre, Formes modulaires de poids 1, Ann. Sci. École Norm. Sup. 7 (1974), 507-530.

5. G. Harder, Chevalley groups over function fields and automorphic forms, Ann. of Math. (2) 100 (1974), 249-306.

6. E. Hecke, Mathematische Werke, Vandenhoeck und Ruprecht, Göttingen, 1959.

7. T. Kubota, Elementary theory of Eisenstein series, Kodansha Ltd., Wiley, New York, 1973.

8. S. Lang, Algebraic number theory, Addison-Wesley, Reading, Mass., 1970.

9. W. Li, Newforms and functional equations, Math. Ann. 212 (1975), 285-315.

10. H. Maass, Lectures on modular functions of one complex variable, Tata Institute of Fundamental Research, Bombay, 1964.

11. J.-P. Serre, Abres amalgames et $\mathrm{SL}_{2}$, Collège de France 1968/1969 (note polycopiées, rédigrées avec la collaboration de $\mathrm{H}$. Bass).

12. A. Weil, Basic number theory, Springer-Verlag, New York, 1967.

13. , On the analogue of the modular group in characteristic p, Functional Analysis and Related Fields, Springer-Verlag, New York, 1970, pp. 211-223.

14. __ Dirichlet series and automorphic forms, Lecture Notes in Math., vol. 189, SpringerVerlag, Berlin and New York, 1971.

Department of Mathematics, Harvard University, Cambridge, Massachusetts 02138

Current address: Department of Mathematics, University of Illinois, Chicago, Illinois 60680 